\title{
Whole genome sequencing of Borrelia miyamotoi isolate Izh-4: reference for a complex bacterial genome
}

\author{
Konstantin V. Kuleshov ${ }^{1,2^{*}}$ (D, Gabriele Margos ${ }^{3^{*}}$, Volker Fingerle ${ }^{3}$, Joris Koetsveld ${ }^{4}$, Irina A. Goptar ${ }^{5}$, \\ Mikhail L. Markelov ${ }^{5}$, Nadezhda M. Kolyasnikova ${ }^{1,6}$, Denis S. Sarksyann ${ }^{4,7}$, Nina P. Kirdyashkina ${ }^{5}$, German A. Shipulin ${ }^{8}$, \\ Joppe W. Hovius ${ }^{4}$ and Alexander E. Platonov ${ }^{1}$
}

\begin{abstract}
Background: The genus Borrelia comprises spirochaetal bacteria maintained in natural transmission cycles by tick vectors and vertebrate reservoir hosts. The main groups are represented by a species complex including the causative agents of Lyme borreliosis and relapsing fever group Borrelia. Borrelia miyamotoi belongs to the relapsing fever group of spirochetes and forms distinct populations in North America, Asia, and Europe. As all Borrelia species B. miyamotoi possess an unusual and complex genome consisting of a linear chromosome and a number of linear and circular plasmids. The species is considered an emerging human pathogen and an increasing number of human cases are being described in the Northern hemisphere. The aim of this study was to produce a high quality reference genome that will facilitate future studies into genetic differences between different populations and the genome plasticity of $B$. miyamotoi.
\end{abstract}

Results: We used multiple available sequencing methods, including Pacific Bioscience single-molecule real-time technology (SMRT) and Oxford Nanopore technology (ONT) supplemented with highly accurate Illumina sequences, to explore the suitability for whole genome assembly of the Russian B. miyamotoi isolate, Izh-4. Plasmids were typed according to their potential plasmid partitioning genes (PF32, 49, 50, 57/62). Comparing and combining results of both long-read (SMRT and ONT) and short-read methods (Illumina), we determined that the genome of the isolate Izh-4 consisted of one linear chromosome, 12 linear and two circular plasmids. Whilst the majority of plasmids had corresponding contigs in the Asian B. miyamotoi isolate FR64b, there were only four that matched plasmids of the North American isolate CT13-2396, indicating differences between B. miyamotoi populations. Several plasmids, e.g. Ip41, Ip29, Ip23, and Ip24, were found to carry variable major proteins. Amongst those were variable large proteins (Vlp) subtype $V|p-a, V| p-\gamma, V \mid p-\delta$ and also VIp- $\beta$. Phylogenetic analysis of common plasmids types showed the uniqueness in Russian/Asian isolates of B. miyamotoi compared to other isolates.

Conclusions: We here describe the genome of a Russian B. miyamotoi clinical isolate, providing a solid basis for future comparative genomics of $B$. miyamotoi isolates. This will be a great impetus for further basic, molecular and epidemiological research on this emerging tick-borne pathogen.

Keywords: Borrelia miyamotoi, Plasmids, Reference genome, Whole genome sequencing, Long-read sequencing

\footnotetext{
*Correspondence: konstantinkul@gmail.com; gmargos1@gmail.com ${ }^{1}$ Central Research Institute of Epidemiology, Moscow 111123, Russia ${ }^{3}$ Bavarian Health and Food Safety Authority, German National Reference Centre for Borrelia, Veterinärstr. 2, 85764, Oberschleissheim, Germany Full list of author information is available at the end of the article
}

(c) The Author(s). 2020 Open Access This article is distributed under the terms of the Creative Commons Attribution 4.0 International License (http://creativecommons.org/licenses/by/4.0/), which permits unrestricted use, distribution, and reproduction in any medium, provided you give appropriate credit to the original author(s) and the source, provide a link to the Creative Commons license, and indicate if changes were made. The Creative Commons Public Domain Dedication waiver (http://creativecommons.org/publicdomain/zero/1.0/) applies to the data made available in this article, unless otherwise stated. 


\section{Background}

Borrelia miyamotoi was first discovered in Ixodes persulcatus in Japan and described in 1995 [1]. Subsequently it was discovered to be occurring sympatrically with $B$. burgdorferi sensu lato in several Ixodes species that also transmit Lyme disease spirochetes. These included Ixodes persulcatus in Eurasia [2-7], I. scapularis [8-11] and I. pacificus [12-15] in North America, and I. ricinus in Europe [16-20]. The prevalence of B. miyamotoi in ticks was found to be usually lower than that of B. burgdorferi s.l. although prevalences of $\sim 15 \%$ have been reported in some regions $[3,7,10,16,17,21,22]$. Rodents have been implicated as reservoir hosts for B. miyamotoi $[23,24]$, but transovarial transmission is also known to occur $[25,26]$ and may contribute to the persistence of this Borrelia in nature.

Despite its co-occurrence with B. burgdorferi s.l. in hard-bodied Ixodes ticks, genetic and phylogenetic analyses showed that $B$. miyamotoi belongs to the clade of relapsing fever (RF) spirochetes [1, 2, 16, 23, 27], which are usually transmitted by soft ticks (Argasidae) or lice. Similar to other relapsing fever species, B. miyamotoi possesses genes encoding variable large proteins and variable small proteins (Vlp and Vsp, respectively) [11, 28, 29]. Vlp and Vsp are expressed during the vertebrate phase of the life cycle of relapsing fever spirochetes. These proteins belong to an antigenic variation system of the spirochetes that permits escape of the hosts' acquired immune response. This can prolong presence of the spirochetes in the blood stream of an infected animal, thus increasing the opportunity of transmission to a vector [30, 31]. Genetic studies on field-collected samples suggested that there is little genetic variability of $B$. miyamotoi isolates within the population of a single tick species, whilst $B$. miyamotoi isolates from different tick species appeared genetically heterogeneous [3, 22]. Thus, it was suggested that the species $B$. miyamotoi consists of Asian, European, North American - West and East Coast - ecotypes/genotypes [2, 8, 16, 32, 33].

The first cases of human disease caused by B. miyamotoi were reported in 2011 in Russia [3]. In that study, 46 cases of B. miyamotoi disease (BMD) were described with clinical manifestations that included fever and an influenza-like illness, with myalgia and arthralgia amongst other symptoms. Since then, several hundred BMD cases were identified in Russia [34, 35]. BMD cases have been reported in Europe and the USA as well, but not with such frequency [2, 36-39]. Cases that were reported from Western Europe often involved immunocompromised individuals, but more recently also immunocompetent persons [40, 41]. The widespread geographic distribution of this emerging human pathogen that can utilize many different vectors and hosts, as well as the different clinical presentation of BMD, varying in clinical significance from asymptomatic infection to severe effects such as meningoencephalitis, imply the need to understand the genetic basis of this diversity.

However, compared to other bacterial genomes, Borrelia genomes are unusually complex, consisting of a linear chromosome and a number of linear and circular plasmids. Plasmid content and structure does not only vary amongst species, but also may vary within species. Thus the assembly of the complete B. miyamotoi genome is a challenging task.

So far, the genome of one B. miyamotoi isolate FR64b of the Asian subtype and four American isolates (CT132396, CA17-2241, LB2001, CT14D4) have been sequenced [11, 14, 33, 42]. However, a long-read sequencing method was used only for the characterization of CT132396. Therefore the number and content of plasmids is not described properly for the other four strains [43].

In the current study, we sequenced the genome of one Russian B. miyamotoi patient isolate. The aim of our study was to produce a high quality genome for $B$. miya$m o t o i$ in order to provide a reference for further studies into the genetic diversity and the genome plasticity of $B$. miyamotoi. To this end, we evaluated several sequencing and bioinformatics methods, as well as several methods for identification and classifying plasmids. We compared and combined different long-read methods (Pacific Biosciences single-molecule real-time technology (SMRT) and Oxford Nanopore Technology (ONT)) and supplemented assemblies with accurate Illumina short-read sequences. The resulting reference genome will help to simplify and improve future genomic analysis of $B$. miyamotoi isolates, in particular to investigate specific genomic features of Asian B. miyamotoi isolates and to identify and investigate virulence and pathogenicity factors.

\section{Results}

\section{PFGE analysis of $B$. miyamotoi lzh-4 strain}

Pulsed-field Gel Electrophoresis (PFGE) analysis revealed a chromosome with a length of $\sim 900 \mathrm{~kb}$ and nine nonchromosomal fragments (potential plasmids) (Fig. 1). The first three non-chromosomal fragments with sizes ranging from $72 \mathrm{~kb}$ to $64 \mathrm{~kb}$ were similar among all Russian B. miyamotoi isolates [44] (data not shown). The remaining bands indicated the presence of additional six plasmids with sizes ranging from approx. $40 \mathrm{~kb}$ to $13 \mathrm{~kb}$. This is probably an underestimate, since it is well known that plasmids with similar sizes or circular plasmids (which may have different migration patterns than linear plasmids) may not be identified by PFGE.

\section{B. miyamotoi strain, genome sequencing and assembly}

In order to obtain a high quality reference genome for comparative genomics of $B$. miyamotoi, the genome of 


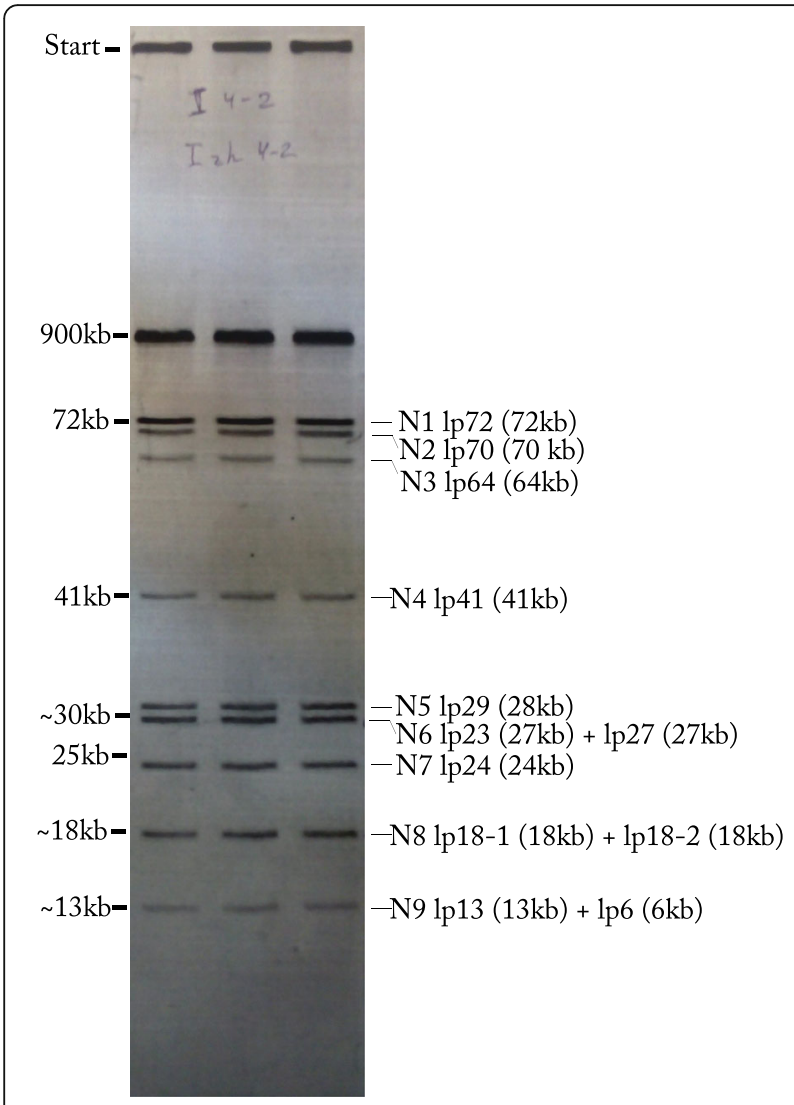

Fig. 1 PFGE pattern of chromosomal and plasmid DNA of $B$. miyamotoi isolate Izh-4 in three independent repetitions. N1-N9 indicate PFGE fragments which were subjected to gel extraction and sequencing via the Illumina platform. The name of plasmids with corresponding length is given on the right site of the gel. It was based on the comparison of assembled contigs from each of the PFGE fragments with the final assembly. Of note, the Ip6 plasmid did not separate in PFGE, no distinct band at that size was visible. This may have been due to insufficient PFGE conditions, as Ip6 sequences were identified in the fragment of $13 \mathrm{~kb}$ together with plasmid Ip13 by direct sequencing

isolate Izh-4 was randomly chosen from available Russian clinical isolates [44] (Additional file 1: Table S1) and sequenced using different sequencing platforms including Illumina MiSeq and HiSeq, ONT MinION, and Pacific Biosciences SMRT. Assemblies of long reads were corrected using long reads (e.g. PacBio with PacBio; ONT with ONT) and subsequently using highly accurate Illumina sequence reads by means of the Pilon pipeline [45].

Using the MinION platform we obtained 129,992 raw reads of an average length of $6.6 \mathrm{~kb}$. After correction and trimming in the Canu v1.7 pipeline the number of long reads decreases to 31,584 with an average length $7.3 \mathrm{~kb}$. The assembly showed 16 contigs with lengths ranging from $900 \mathrm{~kb}$ to $10 \mathrm{~kb}$. Manual validation revealed that two of them - tig00009030 and tig00000013 - were characterized by a specific coverage pattern of ONT reads in two peaks indicating that two separate plasmids were merged. Moreover, the two contigs were $46 \mathrm{~kb}$ and $50 \mathrm{~kb}$ in size, which was not in line with the PFGE analysis (Additional file 2: Figures S1-S3). Therefore, these contigs were split into two contigs and processed as separate plasmids. In addition, three of the resulting 18 contigs were characterized by low long read coverage (2-3x) and had a high similarity level $(\geq 95 \%)$ to other contigs and were therefore removed from further analysis. Finally, two of the 15 remaining contigs were automatically circularized with lengths of $30 \mathrm{~kb}$ and $29 \mathrm{~kb}$. To summarize, using this method, in the end we obtained 15 contigs corresponding to one main chromosome and 14 potential plasmids, with coverage by trimmed reads ranging from $300 x$ to $20 x$ (Table 1 ).

Using the PacBio platform we obtained 312,224 raw reads with an average length of $4 \mathrm{~kb}$. Using 2635 corrected reads with an average length of $8.8 \mathrm{~kb} 20$ contigs were assembled, with a contig length varying from $6 \mathrm{~kb}$ to $906 \mathrm{~kb}$. Three low-coverage contigs, with sequences present in other parts of the genome, were assumed to be assembly artifacts and were removed. Two contigs were manually circularized based on overlapping ends.

Mismatches between ONT and PacBio assemblies were noted and differences to hypothetical lengths of plasmids in PFGE were observed. PacBio unitig\#3 was $68 \mathrm{~kb}$ in size and was not identified in PFGE. It was similar to three separate ONT contigs ( $41 \mathrm{~kb}, 27 \mathrm{~kb}$ and 22 kb) (Additional file 2: Figure S4). Three PacBio unitigs corresponding to an ONT contig of $70 \mathrm{~kb}$ were identified, so ONT contig was mistakenly split into three separate PacBio contigs (Additional file 2: Figure S5). Moreover, two of these PacBio unitigs \#20 ( $38 \mathrm{~kb})$ and \#22 ( $38 \mathrm{~kb})$ were not observed in PFGE. The $64 \mathrm{~kb}$ ONT contig was partially represented in unitig\#10, which was $43 \mathrm{~kb}$ in size (Additional file 2: Figure S6) and also not found in PFGE. These mis-assemblies of PacBio sequences might have been due to a low amount of DNA submitted for sequencing $(1.2 \mu \mathrm{g})$, which was lower than requested by the sequencing service (5$10 \mu \mathrm{g})$ and did not permit BluePippin size selection. Nonetheless, the remaining contigs were similar between PacBio and ONT assemblies. ONT contigs that were split based on coverage analysis were confirmed by PacBio unitigs as separate sequences. Overall, the extracted consensus sequences from PacBio and ONT assemblies (corrected by using highly accurate Illumina reads) resulted in a complete genome consisting of a chromosome of $\sim 900 \mathrm{~kb}$, and 14 putative plasmid contigs, of which two were circular and 12 linear, ranging in length from 6 to $73 \mathrm{~kb}$.

The contigs of the above-described final assembly was also compared with the contigs obtained by direct sequencing of DNA fragments extracted from the agarose 
Table 1 The final composition of B. miyamotoi lzh-4 genome and coverage by long and short reads

\begin{tabular}{|c|c|c|c|c|c|c|}
\hline & $\begin{array}{l}\text { GenBank accession } \\
\text { numbers }\end{array}$ & $\begin{array}{l}\text { Molecule } \\
\text { name }\end{array}$ & Length, bp & $\begin{array}{l}\text { PacBio read coverage before and after } \\
\text { correction in brackets }\end{array}$ & $\begin{array}{l}\text { MinION read coverage before and after } \\
\text { correction in brackets }\end{array}$ & $\begin{array}{l}\text { Illumina reads } \\
\text { coverage }\end{array}$ \\
\hline 1 & СР024390.1 & chromosome & 906,129 & $695 x(16 x)$ & $668 x$ (200x) & $440 x$ \\
\hline 2 & СР024391.1 & plzh4-Ip72 & 73,492 & $1378 x(24 x)$ & $1118 x(300 x)$ & $402 x$ \\
\hline 3 & СР024392.1 & plzh4-Ip70 & 70,072 & $677 x(17 x)$ & $573 x(122 x)$ & $359 x$ \\
\hline 4 & СР024401.2 & plzh4-Ip64 & 64,141 & $321 x(5 x)$ & $365 x(67 x)$ & $262 x$ \\
\hline 5 & СР024393.1 & plzh4-Ip41 & 41,127 & $509 x(11 x)$ & $447 x(54 x)$ & $523 x$ \\
\hline 6 & СР024395.1 & $\begin{array}{l}\text { plzh4-cp30- } \\
1\end{array}$ & 30,091 & $1712 x(26 x)$ & $591 x(162 x)$ & $192 x$ \\
\hline 7 & СР040828.1 & $\begin{array}{l}\text { plzh4-cp30- } \\
2\end{array}$ & 29,490 & $657 x(13 x)$ & $265 x(49 x)$ & $177 x$ \\
\hline 8 & СР024396.1 & plzh4-Ip29 & 28,667 & $1211 x(23 x)$ & $544 x(72 x)$ & $614 x$ \\
\hline 9 & СР024397.1 & plzh4-Ip23 & 27,717 & $528 x(16 x)$ & $329 x(37 x)$ & $504 x$ \\
\hline 10 & СР024398.1 & plzh4-Ip27 & 26,599 & $862 x(17 x)$ & $334 x(20 x)$ & $251 x$ \\
\hline 11 & СР024399.2 & plzh4-Ip24 & 24,033 & $1263 x(18 x)$ & $470 x(78 x)$ & $466 x$ \\
\hline 12 & СР024400.2 & plzh4-Ip18-2 & 18,334 & $1554 x(17 x)$ & $323 x(63 x)$ & $722 x$ \\
\hline 13 & СР024405.2 & plzh4-Ip18-1 & 18,024 & $771 x(14 x)$ & $123 x(49 x)$ & $527 x$ \\
\hline 14 & СР024404.1 & plzh4-Ip13 & 13,410 & $480 x(9 x)$ & $118 x(43 x)$ & $327 x$ \\
\hline \multirow[t]{3}{*}{15} & СР024407.1 & plzh4-Ip6 & 5851 & $578 x(3 x)$ & $138 x(92 x)$ & $625 x$ \\
\hline & & & $\begin{array}{l}\text { Total } \\
\text { reads: }\end{array}$ & $312,224(2625)$ & $129,992(31,584)$ & $2,642,950$ \\
\hline & & & $\begin{array}{l}\text { Mapped } \\
\text { reads: }\end{array}$ & $95 \%(100 \%)$ & $100 \%$ (100\%) & $93 \%$ \\
\hline
\end{tabular}

Raw and trimmed \corrected long reads from MinION and PacBio as well as short reads from Illumina were mapped to the final assembly of Izh-4 genome by mininap2 (https://github.com/lh3/minimap2) with default parameters for each type of reads

gel after separation by PFGE. These contigs were matched using Mummer and visualized by Circos. A number of contigs were produced for the different bands, but only a subset in each band represented the plasmid in question (see Fig. 1 and Additional file 2: Figures S7-S15). For example, for the PFGE fragment N1, 85 contigs were assembled from Illumina short reads, but only one contig of a length of $72,707 \mathrm{bp}$ completely reproduced the lp72 plasmid in the final assembly. Although we were able to identify the majority of linear plasmids by direct sequencing of PFGE fragments, among the collected contigs no sequences corresponding to circular plasmids (cp30-1 and cp30-2) were found. Two of the plasmids, namely lp70 and lp64, were highly fragmented. Many small contig with low k-mer coverage compared to major contigs were observed and were possibly the result of sample contamination during the DNA isolation process.

The final composition of genome is summarized in Table 1. This assembly was deposited in GenBank, BioSample SAMN07572561.

\section{Determination of telomere sequences on the left and right ends of linear replicons}

The genome of isolate Izh-4 of Borrelia miyamotoi contains 13 linear replicons. As palindromic sequences were reported at the ends of linear plasmids in other Borrelia species [46] we searched whether the linear replicons were flanked with palindromic sequences that resemble short telomere structures forming covalently closed hairpins. When analyzing the terminal regions of the assembled chromosome and linear plasmids, terminal nucleotide sequences were identified, which are presented in Table 2. Identical palindromic sequences were found for lp70R and lp18-1 L, lp70L and lp13L, lp64L and $1 p 41 \mathrm{~L}, \mathrm{lp} 29 \mathrm{R} / \mathrm{lp} 24 \mathrm{~L} / \mathrm{lp} 23 \mathrm{R}, \mathrm{lp} 29 \mathrm{~L}$ and $\mathrm{lp} 27 \mathrm{~L}, \mathrm{lp} 24 \mathrm{R}$ and lp18-2 L. The lp6L sequence - although palindromic - might not have been identified properly as there was no "signature" sequence.

Due to the absence of detailed information about telomere sequences for relapsing fever Borrelia, and in particular B. miyamotoi, we can only suppose that there is evidence for the presence of "Box 3" with the consensus motif "WTWGTATA" starting from position 14, as previously described for Lyme disease Borrelia [46-48]. The sequence described as "Box 3" corresponds to a previously annotated conserved region (Box 3 ), which was assumed to be directly involved in interaction with the telomere resolvase ResT $[49,50]$.

\section{Genome content}

Genome annotation of isolate Izh-4 revealed a total of 1362 genes including 31 genes for transfer RNA (tRNA), one cluster of three genes of ribosomal RNA (rRNA) (5S, 16S, 23S) and three genes of non-coding RNA (ncRNA). Out of the 1362 genes, 1222 have been annotated as protein-coding genes. The analysis showed the 
Table 2 Telomere sequences of chromosome and linear plasmids of isolate Borrelia miyamotoi Izh-4

\begin{tabular}{rr}
\hline Location & Direction 5'-3' \\
\hline ChrL & ATATTAAATTAAAAGTATAAACCTATCAACACCA \\
lp72L & AGTTTAAATTTTAATAGTATAAATAACAACTCATTTA \\
lp72R & ATATTTATTTTTTATAGTATAAAATGTCTATAATAAA \\
lp70L & ATGTAATTTGTTTTAATATAAGACTCCTTGAAAAT \\
lp70R & AGTTTATTAGCTATTAGTATAAAGTTAAATTAAAAAT \\
lp64L & AGTTAAATATTGTTTTGTATAATAGACAAGCTTTCAG \\
lp64R & AGTTTATTTGTTATTAGTATAAATCATTGCCCAATC \\
lp41L & AGTTAAATATTGTTTTGTATAATAGACAAGCTTTCAG \\
lp41R & AGTTAATTAGTGTTGTATAATAGACAAGCTTACAC \\
lp29L & TATAATTTGTTAATAGTATAAAGTTTGGCCTTATTT \\
lp29R & AGTTAATTAGTGTGTTGTATAATAGACAAGCTTACAC \\
lp27L & TATAATTTGTTAATAGTATAAAGTTTGGCCTTATTT \\
lp27R & AGTTTAGTTTTTATTAGTATAAATCTACGACAATCTA \\
lp24L & AGTTAATTAGTGTGTTGTATAATAGACAAGCTTACAC \\
lp24R & TATAATTTGTTAATAGTATAGATTGCTTCTTTTTAT \\
lp23L & ATGTAATTTGTTTTTAGTATAAAACTCCTTGAATAAT \\
lp23R & AGTTAATTAGTGTGTTGTATAATAGACAAGCTTACAC \\
lp18-2L & TATAATTTGTTAATAGTATAGATTGCTTCTTTTTAT \\
lp18-2R & AATTTATTAGTTTTTAGTATAAAATTGAAAGGAAAGA \\
lp18-1L & TTTATTAGCTATTAGTATAAAGTTAAATTAAAAAT \\
lp18-1R & AGTTTATTTTTATTAGTATATTGTCTATTTACCTTA \\
lp13L & ATGTAATTTGTTTTTAGTATAAGACTCCTTGAATAAT \\
lp13R & AGTTAAATATTGTTTTGTATAATAGACAAGCATGTCT \\
lp6L ? & TTACTATGTACGAAAATAGCTAAATGCTATATTATGT \\
lp6R & AGTTTTCTTTTTTTTGTACAAAATATAATATAACTA \\
\hline
\end{tabular}

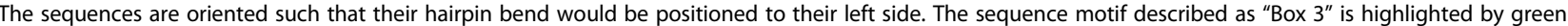
background. The partly identified sequence motif of "Box 3 " is highlighted by yellow background. "?" - indicate telomere sequence which might not have been identified properly

presence of $103(7.5 \%)$ pseudogenes in the Izh-4 genome (Table 3). The majority of pseudogenes were the result of a frameshift. The number of pseudogenes differed between genomic elements and ranged from 0 to 24 . The highest number of pseudogenes was present in two plasmids, lp70 and lp64, and in the chromosome, with 24, 23 and 22 pseudogenes, respectively.

Functional classification of proteins by comparison with previously defined clusters of orthologous groups (COG) showed that approximately $81 \%$ of chromosomal proteins and only $16 \%$ of the plasmid proteins of Izh- 4 could be assigned to 25 different COG categories (RPSBLAST, threshold $E$-value 0.01). This confirms that the chromosome is well conserved. Indeed, a comparison based on COG between the chromosomes of Russian isolates with the previously sequenced genomes of the American (CT13-2396) and Asian (FR64b) genotypes did not reveal significant differences either.

The high percentage of COG-classified proteins localized on some plasmids indicates that some plasmids carry vital genes that likely encode proteins that contribute to basic metabolic processes. For example, according to our analysis plasmid lp41 (41 kb) encodes 12 COGclassified proteins, and the three plasmids lp72, lp70 and lp64 encode 15, 10 and 9 of such proteins, respectively (Table 3). It is worth mentioning that lp41 is the main virulence plasmid carrying and expressing the "main variable surface proteins" (variable major proteins, Vmps) [28].

\section{Borrelia miyamotoi chromosome}

Pairwise sequence comparison of the linear chromosome of Izh-4 with the previously sequenced genomes of FR64b (Japan), CT14D4, LB2001, and CT13-2396 (USA) of B. miyamotoi revealed that the average nucleotide identity (ANI) between chromosomes of Izh-4 and FR64b amounted to $99.97 \%$ and to $97.77 \%$ to isolates from the USA. Whole genome alignment of these chromosomes did not reveal any noticeable genomic rearrangements such as long insertions $\backslash$ deletions, 
Table 3 Gene content analysis of Izh-4 genome

\begin{tabular}{|c|c|c|c|c|c|c|c|c|c|c|}
\hline & $\begin{array}{l}\text { Length, } \\
\text { bp }\end{array}$ & $\begin{array}{l}\text { Total } \\
\text { genes }\end{array}$ & $\begin{array}{l}\text { Total } \\
\text { CDS }\end{array}$ & $\begin{array}{l}\text { COG } \\
\text { classified } \\
\text { genes }\end{array}$ & $\begin{array}{l}\% \text { of COG } \\
\text { classified } \\
\text { genes }\end{array}$ & $\begin{array}{l}\text { Total number } \\
\text { of } \\
\text { pseudogenes }\end{array}$ & $\begin{array}{l}\% \\
\text { pseudogenes } \\
\text { of total genes }\end{array}$ & $\begin{array}{l}\text { Pseudogenes } \\
\text { frameshifted }\end{array}$ & $\begin{array}{l}\text { Pseudogenes } \\
\text { uncompleted }\end{array}$ & $\begin{array}{l}\text { Pseudogenes } \\
\text { with internal } \\
\text { stop }\end{array}$ \\
\hline chromosome & 906,129 & 850 & 791 & 641 & 81 & 22 & 3 & 17 & 2 & 3 \\
\hline lp72 & 73,492 & 72 & 70 & 15 & 21 & 2 & 3 & 2 & 0 & 0 \\
\hline Ip70 & 70,072 & 93 & 69 & 10 & 14 & 24 & 26 & 17 & 5 & 2 \\
\hline Ip64 & 64,141 & 84 & 61 & 9 & 14 & 23 & 27 & 19 & 2 & 2 \\
\hline Ip41 & 41,127 & 35 & 33 & 12 & 36 & 2 & 6 & 2 & 0 & 0 \\
\hline cp30-1 & 30,091 & 42 & 42 & 5 & 11 & 0 & 0 & 0 & 0 & 0 \\
\hline ср30-2 & 29,490 & 42 & 41 & 6 & 14 & 1 & 2 & 0 & 1 & 0 \\
\hline lp29 & 28,667 & 23 & 17 & 1 & 5 & 6 & 26 & 4 & 2 & 0 \\
\hline lp23 & 27,717 & 23 & 20 & 2 & 10 & 3 & 13 & 3 & 0 & 0 \\
\hline |p27 & 26,599 & 31 & 27 & 4 & 14 & 4 & 13 & 1 & 3 & 0 \\
\hline Ip24 & 24,033 & 20 & 16 & 1 & 6 & 4 & 20 & 4 & 0 & 0 \\
\hline |p18-1 & 18,334 & 18 & 10 & 1 & 10 & 8 & 44 & 5 & 2 & 1 \\
\hline Ip18-2 & 18,024 & 13 & 10 & 1 & 10 & 3 & 23 & 1 & 1 & 1 \\
\hline Ip13 & 13,410 & 10 & 9 & 2 & 22 & 1 & 10 & 1 & 0 & 0 \\
\hline Ip6 & 5851 & 6 & 6 & 1 & 16 & 0 & 0 & 0 & 0 & 0 \\
\hline Total: & & 1362 & 1222 & 711 & & 103 & & & & \\
\hline
\end{tabular}

duplications of regions, and translocations, confirming the conservative nature of the $B$. miyamotoi linear chromosome. However, small differences were detected in polymorphisms of tandem repeats (VNTR), single nucleotide polymorphisms (SNPs), and small indels (Additional file 3: Figures S30 - S31 and Table S2). The total number of differences detected among chromosomes was - unsurprisingly - different between isolates from different geographic regions: Izh-4 and isolates from the USA showed an average of 18,563 differences; Izh-4 and the Japanese isolate had merely 122 . The majority of differences were base substitutions. We also identified five sites containing VNTRs (Additional file 3: Figure S30). Such differences may be useful for developing future subtyping schemes for B. miyamotoi clinical isolates.

\section{Plasmid typing by analysis of paralogous gene families (PF) genes}

The identified 14 plasmid contigs and the chromosome of Izh-4 were subjected to an analysis to define the type of partition proteins and to decide on potential names for particular plasmids. In order to identify genes homologous to the plasmid replication/maintenance proteins PF 32, 49, 50, 62 and 57 [51, 52], extracted nucleotide sequences of open reading frames (ORFs), including genes annotated as pseudogenes, from the Izh- 4 genome as well as reference genomes of different Borrelia species were submitted to interproscan annotation and used for comparative phylogenetic analysis (See the Methods section for a more detailed description).
We identified that Izh-4 possessed contigs characterized by different PF genes (Fig. 2). Using a method that was previously described for B. burgdorferi [51], we defined the plasmid types in Izh-4 by investigating the phylogenetic relatedness of PF genes to reference genomes. PF genes $32,49,50,57 / 62$ found on the chromosome and several plasmids (lp72, lp41, lp23, lp6) were phylogenetically closely related and formed monophyletic clades to PF genes corresponding to plasmids of genome CT13-2396 (Additional file 4: Figures S37 S40). Despite the fact that in Izh-4 a plasmid of $27 \mathrm{~kb}$ length had the same PF genes as the plasmid named lp23 in CT13-2396, we choose the same name for these plasmids which is in accordance to plasmid typing in $B$. burgdorferi sl [51]. Notably, PF genes of Izh-4 and FR64b clustered together in more cases than they did with CT13-2396, indicating a closer genetic/genomic relatedness of Russian and Japanese B. miyamotoi isolates than of Russian and North American isolates (including plasmid content).

We found two plasmids - 1p70 and lp64 - that have not previously been described in Borrelia. Each of these plasmids carried several sets of PF genes suggesting that they were formed by fusion of different types of plasmids in the past. Plasmid lp70 of Izh-4 carried two copies of PF32, which phylogenetically clustered with plasmid contigs of FR64b. However, one of the copies showed high similarity to the PF32 of plasmid cp2 of CT132396 (Additional file 4: Figure S37). Plasmid lp64 carried three sets of PF 32, 49, 50,57/62. Of these one cluster 


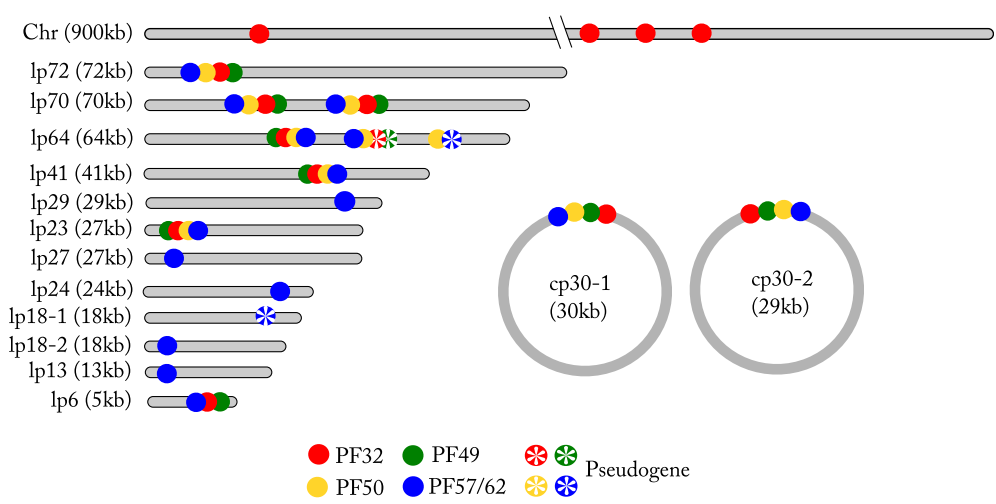

Fig. 2 Schematic representation of the Izh-4 segmented genome with identified PF genes 32, 49, 50,57/62. The order and relative position of these genes on plasmids are displayed

was represented only by PF50 while PF57/62 was a pseudogene and PF32 and PF49 were absent. The other two sets of genes had four PF genes, but one set was characterized by the presence of pseudogenes related to PF 32 and 49 (Fig. 2). Two copies of PF32 of lp64 clustered in different phylogenetic groups and similar copies were found in the FR64b genome. One of the copies of lp64-PF32 is most similar to PF32 located on plasmid pl42 of B. duttonii isolate Ly; the other copy (pseudogene) is most similar to PF32 located on plasmids lpF27 of B. hermsii HS1 and lp28-7 of B. afzelii PKo (Additional file 4: Figure S37).

Plasmids lp29, lp27, lp24, lp18-2, and lp13 possessed only one copy of PF57/62, but the copy in plasmid lp18-1 was a pseudogene of PF57/62. This was consistent with data from previously sequenced genomes [11]. For instance, B. miyamotoi CT13-2396 plasmids lp30, lp20-1, lp20-2 and lp19 have only the PF57/62 gene, and plasmid cp4 only carried a PF50 (Additional file 4: Figure S39, S40). Although the classification of plasmid compatibility types was mainly based on the phylogeny of the PF32 locus, in cases where this locus was absent, we used PF57/62 for plasmid typing. In the phylogeny of PF57/62, plasmids $1 \mathrm{p} 29, \mathrm{lp} 27, \mathrm{lp} 24, \mathrm{lp} 18-2$, and $1 \mathrm{p} 13$ of Izh-4 and other $B$. miyamotoi isolates formed a clade distinct from most other RF and LB species, except for B. hermsii HS1 lpG27. Near identical PF57/62 were found for two pairs of plasmids of Izh-4: plasmids lp29 lp27 and lp18-1 - lp18-2. This could raise the question whether these are indeed different plasmids. However, these pairs of plasmids had no other extended regions of nucleotide similarity (Additional file 3: Figures S33, S34) beyond the PF57/62 locus, indicating they are two different pairs of plasmids. PF57/62 of plasmid lp13 clustered together with the PF57/62 of lp30 of CT13-2396 and a gene located on a plasmid contig (CP004259.1) of FR64b. The PF57/62 of Izh-4 lp24 was nearly identical to a homologous gene located on a plasmid contigs
(CP004252) of FR64b. It should be noted that clustering of plasmids based on PF32 genes correlates with groups of plasmids based on PF57/62 clustering, indicating a similar evolutionary patterns between PF32 and PF57/ 62. Since we did not identify variants of the PF57/62 genes of previously sequenced $B$. miyamoto $i$ genomes that would be close enough to the PF57/62 genes of the Izh-4 genome, we decided to establish the names of plasmids based on their length.

The analysis allowed us to identify only two circular plasmids, each of which was approximately $30 \mathrm{~kb}$ in length. The percentage of identity between them was $79 \%$. The set and relative position of ORFs between these plasmids was collinear, with the exception of the variation in the number of Mlp genes (cp30-1 had two genes, cp30-2 had one gene) and inversion of the gene cluster of PF 32, 49, 50, 57/62. Both plasmids are characterized by the presence of genes encoding PBSX phage terminase large subunit, site-specific integrase, indicating a relationship to prophage-related plasmids [53-55]. In addition, both circular plasmids are characterized by the presence of a complete set of PF 32, 49, 50, 57/62 genes. According to the phylogeny of the PF32 genes, these two plasmids belong to different phylogenetic clusters. The PF32 gene of plasmid cp30-1 was more closely related to the PF32 gene localized on plasmids pl28 ( $B$. duttonii Ly) and lp28-8 (B. afzelii PKo). In turn, the PF32 gene of plasmid cp30-2 was phylogenetically closest related to the PF32 gene localized on plasmid lpT28 of B. hermsii HS1.

\section{Organization of the Ip41 virulence plasmid}

Plasmid lp41 appears to play a pivotal role in virulence of $B$. miyamotoi by expressing the Vmps, which enable the bacteria to escape the host immune system during infection [28]. We performed a comparison of lp41 plasmids using BLASTn analysis between Izh-4 and earlier sequenced isolates of B. miyamotoi from USA (LB-2001 
and CT13-2396) and Asia (FR64b). This analysis revealed a high degree of similarity in the relatively conserved 3 'and 5' regions flanking the variable region of the Vmp genes (Fig. 3). Izh-4 carries a gene encoding the Vlp- $\delta$ protein (locus tag: CNO09_05195) after the expression site, while genomes FR64b and CT13-2396 carry Vlp- $\gamma$ (BOM_1113, AXH25_04655) (Fig. 4) and LB-2001 carry Vsp1 (I871_B20) (Fig. 5).

Some minor $800 \mathrm{bp}$ insertions were detected at the left-end of lp41plasmids between pairs of isolates: FR64b - Izh-4 and CT13-2396 - LB-2001 (data not shown). At the same time, the number and order of the Vmp genes were unique for each of the isolates (partially shown in Fig. 3 and Fig. 6). In addition, single nucleotide variations as well as a 138 bp deletion in an intergenic region before the expression site were detected in both Asian genomes, Izh-4 and FR64b, in comparison to CT132396 and LB-2001 (Additional file 3: Figure S35). This might be a marker for differentiation of lp41 plasmids of Asian and American genotypes. Importantly, the organization of the sequence expression site did not differ between $B$. miyamotoi isolates, the nucleotide composition of the Ribosome Binding Site (RBS), the "-10", and " -35 " sites were $100 \%$ identical (Additional file 3: Figure S35, bottom), which could be very helpful in identifying the expressed Vmp [28].

\section{Intragenetic diversity of variable large proteins and variable small proteins}

All Izh-4 nucleotide sequences of genes and pseudogenes were searched to assess whether they belonged to the family of lipoproteins in the InterPro database. In total, we found 39 genes encoding variable large proteins (Vlp), nine of them were pseudogenes, and 15 genes encoding variable small proteins (Vsp), including five pseudogenes. Vlp and Vsp genes were clustered in an island manner and were mostly located on plasmids lp41, lp29, lp23 and lp24. Some single Vsp genes were located on lp64, lp18-2 and lp13 plasmids (Fig. 6).

Phylogenetic analysis of the extracted Vlp genes and pseudogenes of four B. miyamotoi genomes showed that Vlp genes of Izh-4 formed well supported clades: four clades of Vlp- $\delta$ (20 genes), Vlp- $\gamma$ (13 genes), Vlp- $\alpha$ (five genes) families and one gene on lp29 plasmid corresponded to Vlp- $\beta$ (Fig. 4). The closest homologues to Vlp- $\beta$ at $78 \%$ amino acid identity were identified in the genomes of $B$. crocidurae DOU (AHH07120.1) and $B$. hermsii (WP_064536660.1). Notably, Vlp- $\beta$ genes were not described in the genomes of B. miyamotoi LB2001 [28], however, similar genes were present in the genome of CT13-2396 (AXH25_04965) and the partially sequenced genome of FR64b genome (BOM_1386) (Fig. 6, lower purple branch).

Phylogenetic analysis of the extracted Vsp genes did not show any patterns of clustering (Fig. 5). However, comparing 14 of Vlp and 4 Vsp genes showed that they are present in two identical copies located on plasmids lp41 and lp23. A BLAST analysis of nucleotide sequences of these plasmids showed that the right parts of plasmids lp41 and lp23 were identical, with the same order of Vlp and Vsp genes and its pseudogenes (Additional file 3: Figure S36). Pairwise comparison of plasmids containing clusters of these genes did not reveal any similarities like the one found between lp41 and lp23. Such right-end similarity of lp41 and lp23 was also detected in CT13-2396.

\section{Comparison of plasmid sequences among B. miyamotoi isolates}

To explore the plasmid similarity between different $B$. miyamotoi isolates, we compared the nucleotide sequences of the three isolates CT13-2396, FR64b and Izh-4 (Additional file 2: Figure S15 - S29). We chose these isolates since for CT13-2396 an almost complete genome and for Izh-4 a completed genomes were

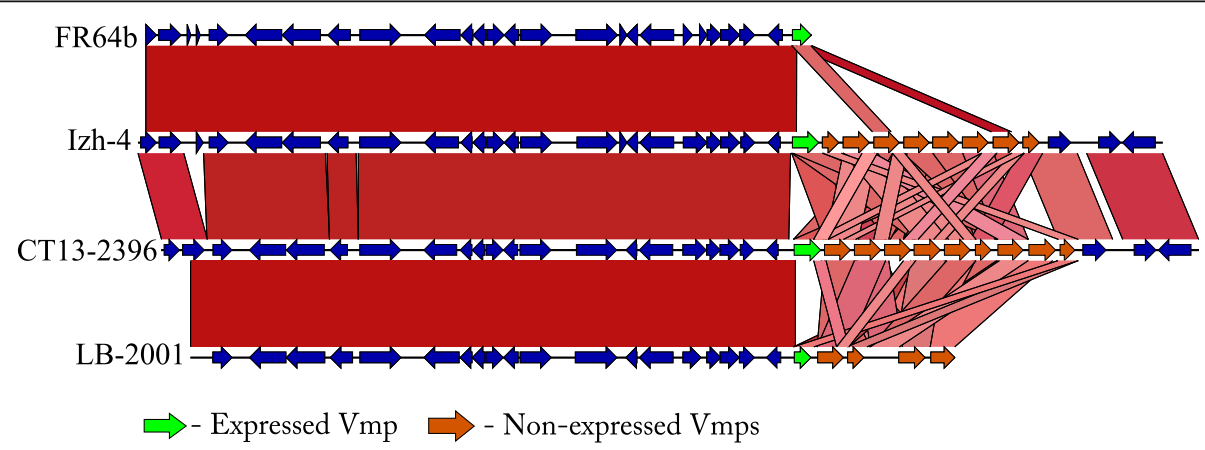

Fig. 3 Comparison of the nucleotide sequences of the virulence plasmid Ip41 of B. miyamotoi isolates originating from North America (LB-2001, CT13-2396), Japan (FR64b), and Russia (Izh-4). Blocks that are colored in red (range of percent identity 100-70\%) indicate similar areas between plasmids. The arrows indicate the genes and direction of ORF. The Vmp block of genes is represented by the genes immediately after the expression site - expressed Vmp (light green arrow) and non-expressed Vmp genes (orange arrows). Other ORFs are shown as blue arrows 


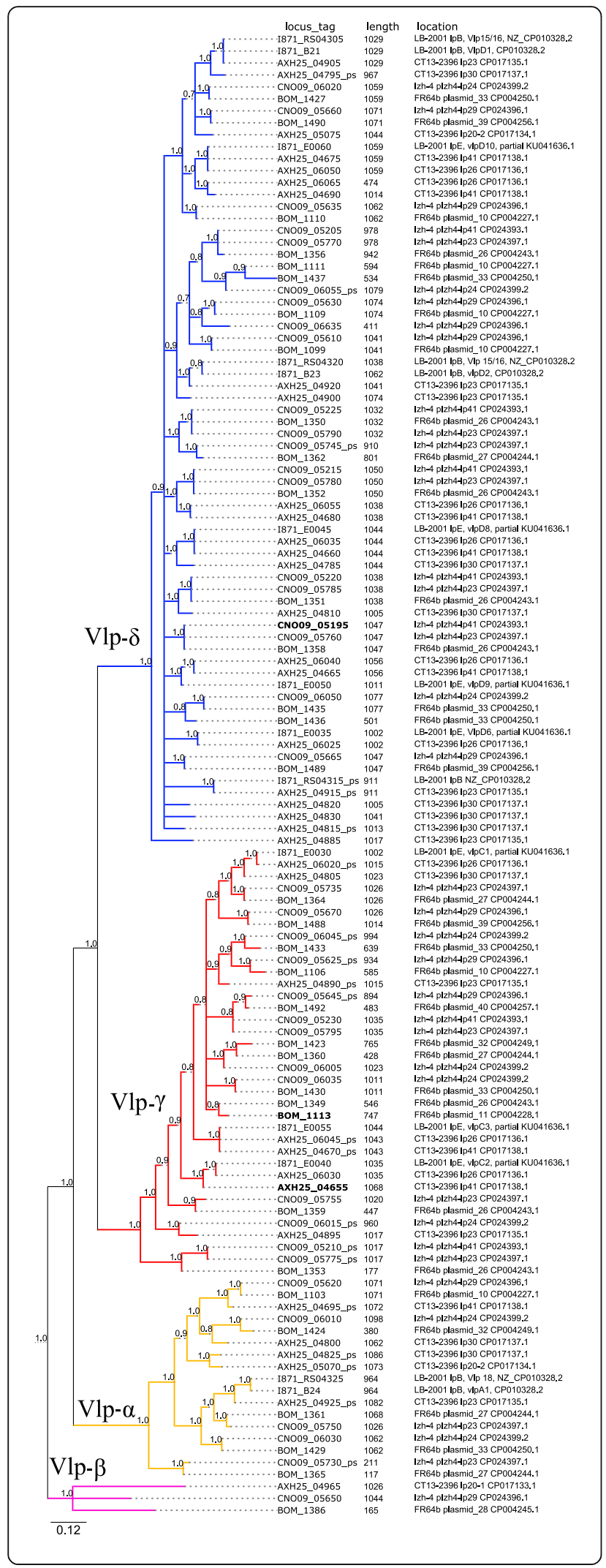

Fig. 4 Phylogenetic diversity of Vlp genes in Izh-4, FR64b, CT132396 and LB-2001 genomes. Different colors of nodes indicate different VIp-subfamilies: yellow - VIp-a, red - VIp- $\gamma$, blue - VIp- $\delta$, purple - VIp- $\beta$. ORFs marked by bold font are genes located after the expression site. Locus tags of pseudogenes are marked by the postfix “_ps". The tree was constructed based on pairwise alignment of nucleotide sequences of ORFs/pseudogenes which contain domains corresponding to the lipoprotein_2 family (PF00921) or the Variable surface antigen VIsE superfamily (SSF74748). VIp- $\beta$ genes were used as outgroup to root the tree

available and for FR64b a draft genome with 50 contigs was accessible in GenBank. Within these three genomes, we found four common plasmids with high nucleotide similarity: lp72, lp41, lp23 and lp6 (Table 4). Plasmids lp70, lp64, lp27, and lp13 of Izh-4 were only present in the Asian FR64b genome, but absent in the North American isolate CT13-2396. Plasmids cp30-1, cp30-2, lp29, lp24, lp18-1 and lp18-2 were partly present in the F64b genome, and absent in CT13-2396.

\section{Phylogenetic analyses \\ Phylogeny of Borrelia spp. based on chromosomal genes}

To understand the relationships of isolate Izh-4, North American and Asian B. miyamotoi isolates as well as to other Borrelia species, we performed a phylogenetic analysis of the newly sequenced genome (Izh-4) and Borrelia genomes deposited in GenBank (Additional file 1: Table S1). To date, these genomes comprised completed chromosomes and/or several completed plasmids (lp73, lp41, lp23 and lp6). The phylogenetic tree was reconstructed using a concatenated alignment of nucleotide sequences of 249 core genes localized on the chromosome (minimum percent identity for BLASTp 70\%) and identified during the process of protein clustering among all Borrelia genomes. This phylogenetic analysis showed that $B$. miyamotoi forms a monophyletic clade inside the relapsing fever group and was split into two lineages belonging to the Asian and American genotype. The Asian lineage includes the Izh-4 and FR64b from Japan (Fig. 7a).

For a more detailed analysis, i.e. to determine intraspecific differences among $B$. miyamotoi isolates, we conducted a reciprocal BLASTp search for core genes, but now only within the species B. miyamotoi. As a result, 719 orthologous genes were identified (minimum percentage identity for BLASTp 80\%) (Fig. 7b).

Mean SNP-distances (in concatenated alignment of core genes) between isolates from Northeast America (CT13-2396, CT14D4, LB-2001), Japan (FR64b) and Russia (Izh-4) were as follows: Northeast American Russian - 13,767 SNPs, Northeast American - Japanese - 13,776 SNPs, and Russian - Japanese - 36 SNPs. Among the three Northeast American isolates six SNPs were found. 


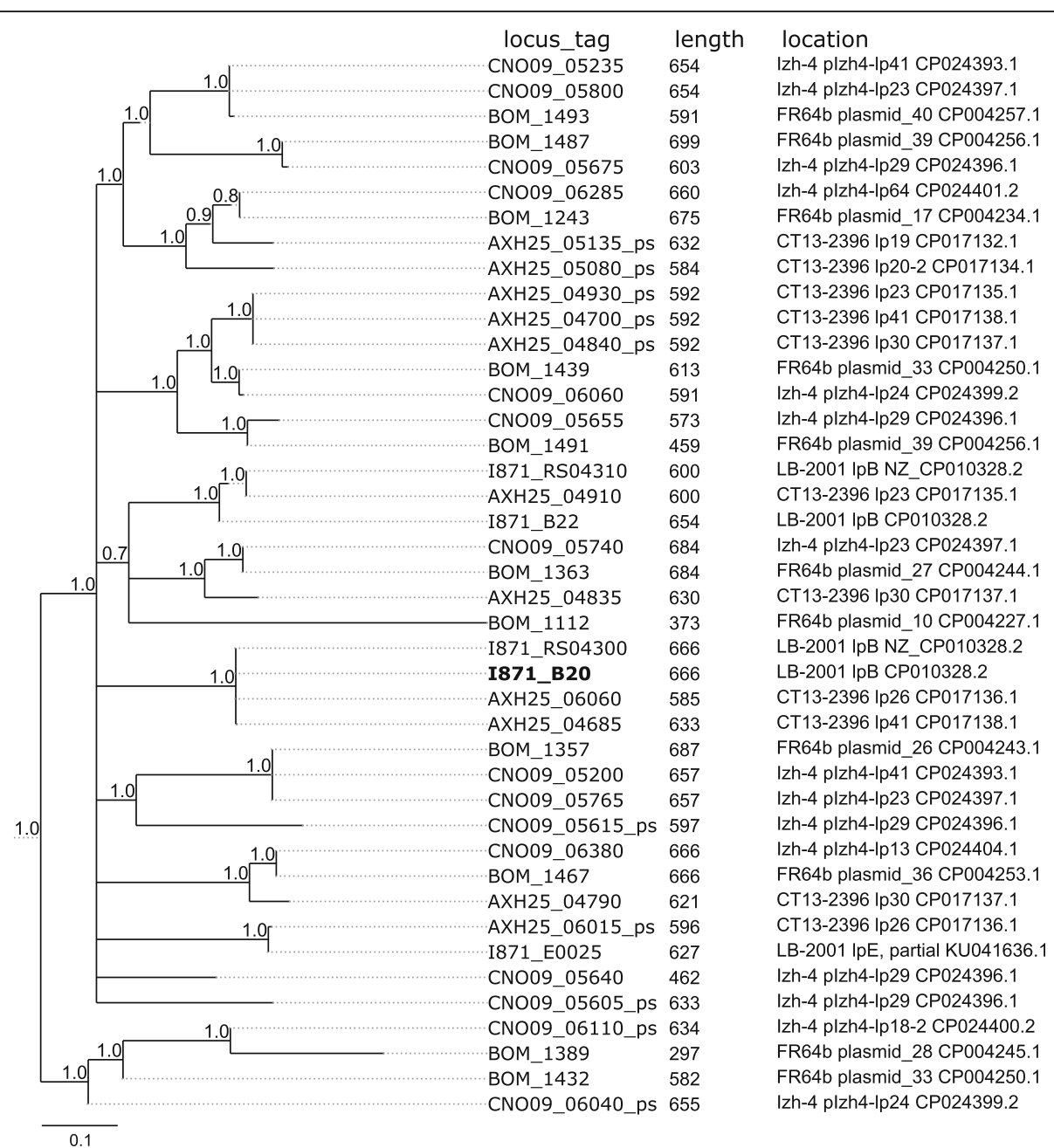

Fig. 5 Phylogenetic diversity of Vsp genes in Izh-4, FR64b, CT13-2396 and LB-2001 genomes. ORFs marked by bold font are genes located after the expression site. Locus tags of pseudogenes are marked with the postfix "_ps". The tree was constructed based on pairwise alignments of nucleotide sequences of ORFs/pseudogenes which contain domains corresponding to the lipoprotein_6 family (PF01441) or the outer surface protein C (OspC) superfamily (SSF63515). The tree was midpoint rooted

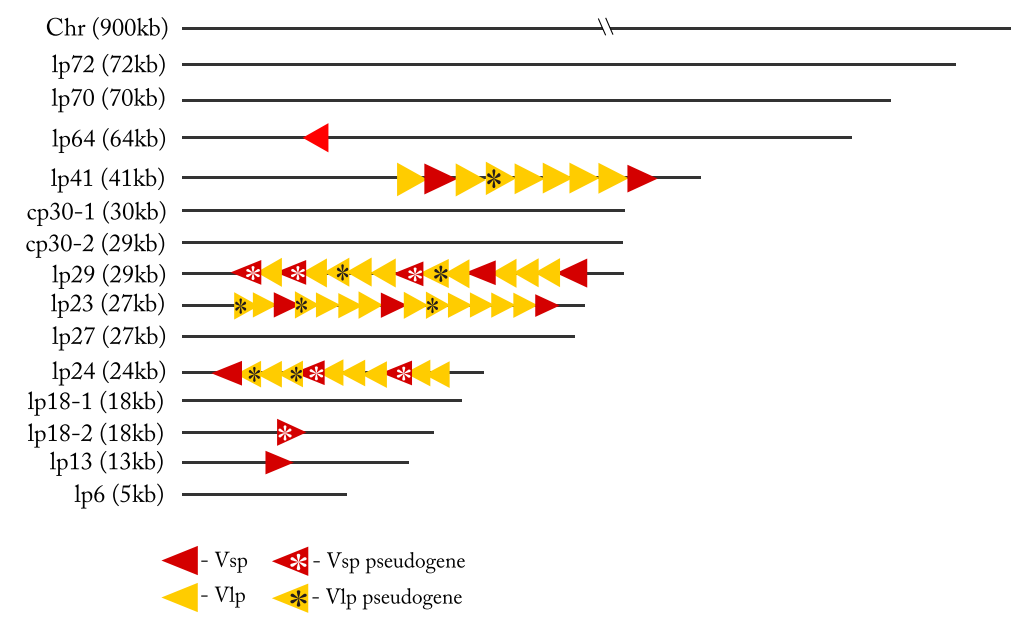

Fig. 6 Number and location of Vmp genes in the Izh-4 genome 
Table 4 Plasmid comparisons of B. miyamotoi strains

\begin{tabular}{lll}
\hline $\begin{array}{l}\text { Izh-4 plasmids as reference } \\
\text { sequences }\end{array}$ & $\begin{array}{l}\text { Matched plasmids of CT13-2396 genome to Izh-4 } \\
\text { plasmids }\end{array}$ & Matched contigs of FR64b genome to Izh-4 plasmids \\
\hline plzh4-Ip72 & Ip72 & CP004218 \\
plzh4-Ip70 & absent & CP004223, CP004233, CP004231, CP004221 \\
plzh4-Ip64 & absent & CP004238, CP004234, CP004222, CP004240, \\
plzh4-Ip41 & Ip41 & CP004239 \\
plzh4-cp30-1 & absent & CP004228 \\
plzh4-cp30-2 & absent & partially covered by CP004247 and CP004219 \\
plzh4-Ip29 & absent & partially covered by CP004220, CP004230 and \\
plzh4-Ip23 & CP004229 \\
plzh4-Ip27 & partially covered by CP004227 and CP004256 \\
plzh4-Ip24 & Ip23 & CP004244, CP004243 \\
plzh4-Ip-18-2 & absent & CP004235 \\
plzh4-Ip18-1 & absent & partially covered by CP004250 \\
plzh4-Ip13 & absent & partially covered by CP004245 \\
plzh4-Ip6 & absent & partially covered by CP004251 \\
\hline & absent & CP004253
\end{tabular}

\section{Discussion}

Genetically B. miyamotoi has been divided into distinct populations, with population boundaries apparently determined by vector association [2, 8, 16, 32, 33]. To investigate genetic differences in $B$. miyamotoi populations, comparative genomics should be employed, but this requires high quality reference genomes [56]. Although several B. miyamotoi draft genomes from North America, Russia and Japan exist [11, 14, 42, 44], a completed genome has not been finished for the Asian genotype of B. miyamotoi. Therefore, to provide a basis for comparative genomics studies, here we assembled a reference genome for the Russian B. miyamotoi isolate Izh4 using currently available long-read and short-read technologies.

For some bacteria the assembly of completed genomes had been reported using Nanopore sequencing as sole method [57] or combining long-read with Illumina short-read technology [43, 58, 59]. Initial assemblies combining Illumina and Nanopore reads of B. miyamotoi genomes from Russia gave unsatisfactory results, as not all genomic elements (plasmids) were properly assembled [44]. Similar issues were encountered before with B. miyamotoi genomes, i.e. some plasmids were incompletely assembled, even though long- (PacBio SMRT) and short-read (Illumina) methods were combined [11]. We therefore sequenced one of the available Russian isolates, Izh-4, using PacBio SMRT technology (in addition to ONT) and compared contigs obtained using the two long-read technologies with results obtained by PFGE. In addition, for accuracy, consensus sequences were generated using Illumina sequence reads.
This strategy resulted in a completed reference genome for isolate Izh-4 consisting of one linear chromosome of $906 \mathrm{~kb}, 12$ linear plasmids ranging in size from 6 to 72 $\mathrm{kb}$ and two circular plasmids of about $30 \mathrm{~kb}$.

Annotation of the genome of B. miyamotoi isolate Izh4 revealed a total of 1362 ORFs with 37 encoding RNA. The linear chromosome was predicted to encode 850 ORFs and the high level of conservation of the linear chromosome was evidenced by the majority of these loci (81\%) being functionally classified as COG. Alignment and comparison of the chromosome with other Russian isolates (data not shown) and isolates from Japan (FR64b) and North America (LB-2001, CT13-2396, CT14D4) confirmed the conservation of the main chromosome. There were neither major nor minor rearrangements nor insertions/deletions observed in this analysis. As expected, similarity of chromosomes was more pronounced among the Russian and Japanese isolates with fewer SNPs and higher ANI values (ANI 99.9\% compared to $97.7 \%$ between North American and Russian isolates). SNP values between Russian/Asian and North American isolates were 1000 or 300 fold higher, respectively, than between Asian and Russian isolate.

There was considerably more variation in the plasmids fraction, especially between isolates from different continents. For isolate Izh-4, 12 linear and two circular plasmids were identified. Compared to the chromosome, the percentage of functionally COG classified genes ranged from 22 to $5 \%$, suggesting many more CDS with unique or unknown function in the plasmid fraction. Plasmids with the highest percentages of pseudogenes included lp18-1 (44\%), lp64 (27\%), lp70 (26\%), lp18-2 (23\%), and 

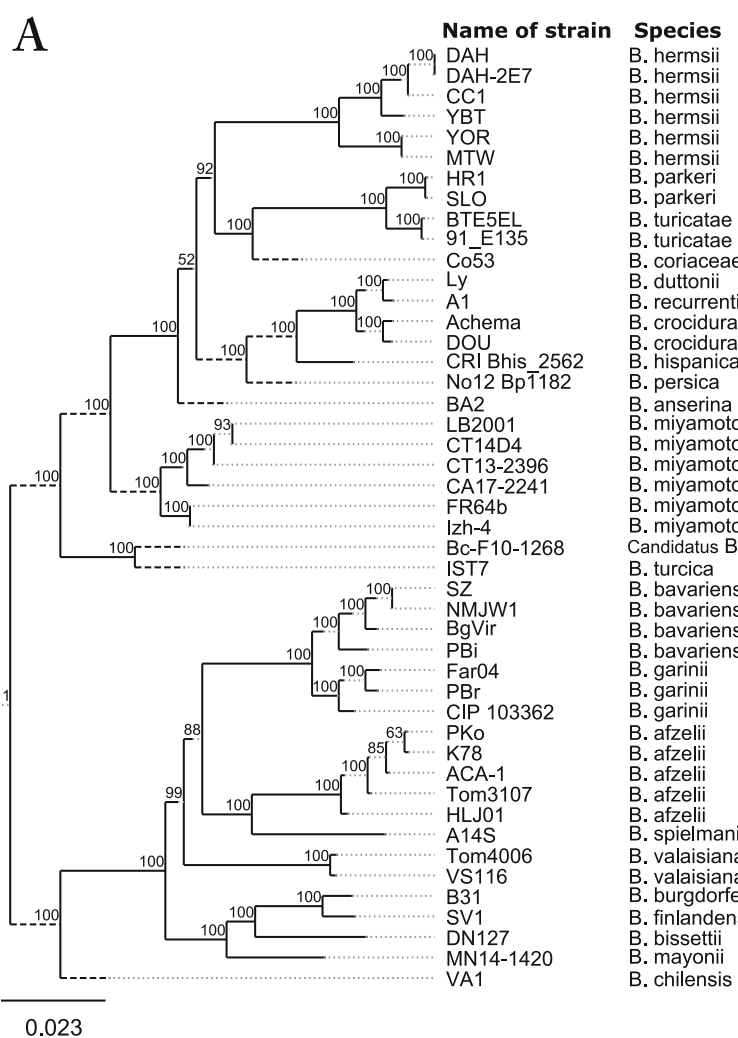

B. hermsii

B. hermsii

B. hermsii

B. hermsii

B. hermsii

B. parkeri

B. turicatae

B. turicatae

B. coriacea
B. duttonii

B. duttonii
B. recurrentis

B. crocidurae

B. crocidurae

B. hispanica
B. persica

B. anserina

B. miyamotoi

B. miyamotoi

B. miyamotoi

B. miyamotoi

B. miyamotoi

Candidatus B. tachyglossi

B. turcica

B. bavariensis

B. bavariensis
B. bavariensis

B. bavariensis

B. garinii

B. garinii

B. garinil

B. afzelii
B. afzelii

B. afzelii

B. afzelii

B. afzelii

B. spiemanii

B. valaisiana

B. burgdorferi

B. finlandensis

B. bissettii

B. mayonii

B. chilensis

Geographical region of isolation

Washington, USA

USA, Spokane

California, USA

USA

USA

USA

USA

Texas, USA

USA

Tanzania

Ethiopia

Mauritania

Marocco

Khiva, Uzbekistan

USA

Connecticut, USA

Connecticut, USA

Connecticut, USA

California, USA

Japan

zhevsk, Russia

Queensland, Australia

Turkey

Heilongjiang, China

nner Mongolia, China

Tomsk, Russia

Germany

Faroe Islands

Germany

Paris, France

Germany

Austria

Sweden

Heilongjiang, China

The Netherlands

Tomsk, Russia

Switzerland

New York, USA

Finland

California, USA

Minnesota, USA

Chile
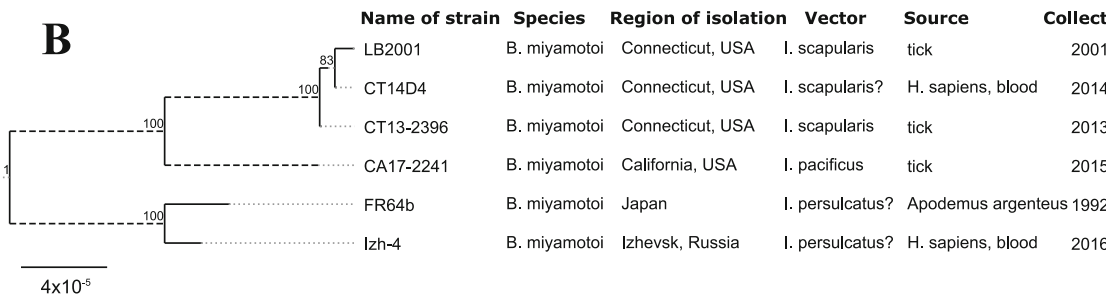

Fig. 7 a Phylogenetic tree of Borrelia species based on the concatenated alignment of nucleotide sequences of 249 core genes located on the chromosome. Borrelia miyamotoi clusters with relapsing fever species. $\mathbf{b}$ Phylogenetic tree of B miyamotoi strains based on concatenated alignment of nucleotide sequences of 719 core genes. A maximum likelihood tree was constructed using RAxML software using a nucleotide substitution model with a gamma distribution of variable positions $(\mathrm{GTR}+\Gamma$ ). The resulting tree was midpoint rooted using Figtree (http://tree.bio.ed.ac.uk/software/ figtree/). Long branches shown not according to scale are indicated by dashed lines. Scale bar indicates substitution rates

lp24 (20\%). In PFGE plasmids with different sizes were observed for the additional five Russian isolates (i.e. Izh5, Izh-14, Izh-16, Yekat-1 and Yekat-6, data not shown), suggesting that the high conservation observed for the $B$. miyamotoi chromosome does not extend to the plasmid fraction. To better understand the evolutionary relationship of plasmids, we employed a previously described method that focused on plasmid replication/maintenance proteins [51, 52].

Since plasmids in Borrelia can vary considerably in size and may undergo intra-specific rearrangements, problems of identifying plasmids due to the similarity of the genomic content exist $[51,60]$. In addition, the transition of plasmids from circular to linear or vice versa makes identifying and classifying plasmids within and between Borrelia species challenging. The importance of plasmid typing is underpinned by the fact that many genes involved in virulence and pathogenicity of Borrelia are plasmid-borne.

Plasmids in the Lyme borreliosis group of spirochetes have been typed and classified using a family of paralogous proteins described as plasmid replication/ maintenance proteins [52, 60, 61]. Due to PF32 being homologous to the ParA protein in other bacteria, this group of proteins - termed PF32, PF49, PF50, and PF57/ 62 - were used to determine the plasmid compatibility type, although in Borrelia PF32 may not fulfil this role $[52,61]$. In the different plasmids, one or several sets of these protein coding genes were found while in others only subsets of these molecules were identified. This is 
consistent with data from previous studies which showed that for Lyme borreliosis spirochetes or B. miyamotoi isolate CT13-2396 some plasmids carried only the PF57/62 gene [11, 51, 52]. Phylogenetic analyses of these paralogous gene family allowed us to identify plasmids of the same compatibility type (i.e. plasmids with $\mathrm{PF}$ genes from the same clade) in draft genomes of North American and Asian B. miyamotoi isolates. Perhaps not surprising, given the greater genetic similarity on the chromosome and the genetic homogeneity of $B$. miyamotoi populations in the same vector species [22, 32 ], more contigs with the same compatibility types of plasmids were found in the Japanese isolate FR64b than in the Connecticut isolate CT13-2396. In the latter isolate four plasmids representing compatibility types to Izh-4 were identified: lp72, lp41, lp23, and lp6. Interestingly, two of these plasmids, lp41 and lp23, contained Vlp and Vsp genes in both isolates. In total in CT132396, 23 ORF encoding Vlp proteins were identified in five plasmids whilst in Izh-4 four plasmids were found encoding a total of $38 \mathrm{Vlp}$ (including 9 pseudogenes).

The characterization of other Russian, North American and European B. miyamotoi isolates - using the technological and bioinformatic platform shown in the current study - is underway. This will confirm the effectiveness of methodology and help to clarify the evolutionary history of Borrelia and to link the genetic peculiarities of B. miyamotoi with epidemiological, microbiological, immunological and clinical features of this emerging infection.

\section{Conclusion}

We have assembled a high quality reference genome for a Russian isolate of B. miyamotoi and this required employing reads from two long-read and a short-read sequencing technologies. This provides a basis for further investigations to identify similarities / differences between B. miyamotoi isolates from different geographical populations and how these may be related to differences in virulence and human pathogenicity.

\section{Methods}

\section{Borrelia miyamotoi isolates and their cultures}

Strain Izh-4 was isolated from blood of Russian patient with acute BMD in Izhevsk City in 2016 and cultured in MKP-F medium [62]. DNA was extracted (see the sections below for the different methods used) from about $10^{9}$ spirochetes after the third in vitro passage.

\section{Plasmid DNA separation by pulsed-field gel electrophoresis (PFGE)}

A standard operating procedure for PulseNet PFGE for Enterobacteria [63] was used with modifications
(Additional file 1: Method S1). Nine extrachromosomal fragments ranging from 13 to $73 \mathrm{~kb}$ were cut out of the gel and dissolved in Agarose Dissolving Buffer (Zymoresearch), DNA was extracted and subjected to Illumina sequencing.

\section{Whole genome sequencing and data analysis Illumina sequencing}

Total DNA was extracted from borrelia suspension using the DNeasy Blood \& Tissue Kit for sequencing using a MiSeq platform. A NexteraXT DNA Library Kit (Illumina, USA) was used for library preparation. DNAlibraries were sequenced using a 500-cycle V2 reagent kit on a MiSeq (Illumina, USA). Low quality reads and adapter sequences were removed from the Illumina reads by BBTools [64]. Assembly was performed by SPAdes-3.9.0 [65].

\section{MinION sequencing and data analysis}

MinION sequencing (Oxford Nanopore Technologies, UK) was performed by ZF-Genomics, Leiden, The Netherlands. Total DNA of isolate Izh-4 was extracted using the Qiagen Tip-100 prep (Qiagen, Germany). The Native Barcoding Kit 1D (EXP-NBD103) was used together with the Ligation Sequencing Kit (SQK-LSK108) to prepare a Nanopore sequencing library from total DNA. A R9.4 MinION flow cell was used for sequencing. Base calling of MinION sequences was performed using Albacore v1.1.0; adapters were removed by Porechop [66]. Canu v1.7 was used for correction, trimming and de novo assembly of ONT long reads with default parameters and a genome size of $1.6 \mathrm{Mb}$. Following assembly each contig was inspected for the presence of long inverted repeats at the ends or end to end overlaps using dot plot analysis implemented in FlexiDot [67]. The revealed overlaps were manually verified by alignment using Mafft v7.271 [68] with subsequent removal. Automatic circularization was performed by an APC (A Perfect Circle) script [69] with manual verification. The draft genome assembly was polished by two steps of correction. First, we mapped corrected ONT reads to contigs with Nanopolish [70]. Then we corrected the obtained consensus sequence by mapping Illumina pair-end reads using Pilon v1.22 [45]. Following read mapping the consensus sequences were extracted for further analysis.

\section{PacBio sequencing and data analysis}

DNA of isolate Izh-4 was submitted to WGS using SMRT sequencing on the Pacific BioScience Technology platform. The sequencing service was provided by the core facility located at the Norwegian Sequencing Centre (NSC) (www.sequencing.uio.no). DNA was extracted from $64 \times 10^{9}$ cells using a Maxwell ${ }^{\circ} 16$ and a Maxwell LEV Blood DNA kit (Promega, Germany). The $20 \mathrm{~kb}$ 
library preparation protocol was employed. Size selection of the final library was performed using $0.4 \mathrm{x}$ Amp beads. The library was sequenced on a Pacific Biosciences RS II instrument using P6-C4 chemistry with $360 \mathrm{~min}$ movie time, two SMRT cells were used for sequencing due to poor loading. De novo assembly was performed using hierarchical genome assembly process (HGAP v3, Pacific Biosciences, SMRT Analysis Software v2.3.0) with default parameters (expected genome size $1.6 \mathrm{Mb}$, minimum target coverage 15X). RS_Resequencing.1 software (SMRT Analysis version v2.3.0) was used to map SMRT reads back to sequences in order to correct contigs after assembly clean-up. PacBio contigs were polished by mapping Illumina pair-end reads using Pilon v1.22.

\section{Determination of the terminal sequences (telomere sequences) of linear replicons}

Determination of the terminal sequences (telomere sequences) of the chromosome and linear plasmids was carried out. For identification of telomere sequences (which are represented as palindrome sequences at the right and left ends of linear contigs) de novo assembled PacBio and ONT contigs were used. Each of the linear elements was aligned against itself and dot plot analysis was performed. For each alignment, we determined the coordinates of the breakpoints determined using dot plot analysis, see (Additional file 5: Figure S41). We cut the nucleotide sequence $200-1000 \mathrm{bp}$ above and below the breakpoint and checked for the presence of palindrome sequences using the Einverted tool of the Emboss package [71]. If palindromes were not detected in a contig by dot plot analysis we mapped the trimmed and preassembled PacBio reads onto this contig and used the part of reads spanning the edge of the left or right ends of the contig and analyzed them in Einverted tool.

\section{Bioinformatics analysis}

\section{Genome sequences used for comparative analysis}

A Table with all isolates used in this study for plasmid typing, comparative genomics and phylogeny is shown in Supplementary information (Additional file 1: Table S1).

\section{Calculation of nucleotide identity between B. miyamotoi chromosomes}

The average nucleotide identity (ANI) between $B$. miyamotoi chromosomes was calculated using the Pyani tool [72] with a BLAST method to align $1020 \mathrm{nt}$ fragments of the input sequences.

\section{Determination and visualization of similarity between genomes and plasmids}

We used Circos v0.69-5 [73] and Mummer v3.0 [74] to show similarities between different plasmids, contigs or different assemblies. For comparison contigs were aligned all versus all and the results of the alignment were visualized using the Circos tool. Only matched regions with more than $90 \%$ identity were taken into account.

To identify differences in the nucleotide sequences of B. miyamotoi chromosomes of various isolates NucDiff [75] was utilized.

To determine whether differences may exist in the virulence plasmid lp41 of different isolates, annotated sequences of lp41 plasmids of B. miyamotoi isolates were visualized and compared in Easyfig software [76].

\section{Genome annotation and designation of plasmid types}

Annotation of assembled contigs was performed using a local version of the NCBI Prokaryotic Genome Annotation Pipeline [77]. To identify the plasmid type of particular contigs we used a principle suggested by Casjens et al. [51] that is based on comparison of paralogous gene families (PF) 32, 49, 50, 62 and 57. The name (nomenclature) that we give to particular contigs (plasmids) is based on the relationship of PF genes identified in those contigs with analogous genes/proteins of previously designated plasmids in a set of reference genomes of different Borrelia species with high-quality annotation. We inferred the relationship of plasmids found in our study by comparison to previous naming schemes of PF [51] and phylogeny. We searched databases of protein sequences by using InterProScan software to find specific PF proteins in annotated genomes such as the Conserved Domains Database (CDD) [78], Protein Families database (Pfam) [79], database of structural and functional annotation for all proteins and genomes (SUPERFAMILY) [80]. For example, PF32 was found to be homologous to proteins in the CDD database with accession numbers cd02038 and cd02042, PF49 is related to PF01672 in Protein Families database, PF50 relates to PF02890, and PF57/62 to PF02414.

Our comparative analysis included the following steps. (1) We extracted all ORFs' nucleotide sequences, including ORFs which were identified as pseudogenes using the NCBI Prokaryotic Genome Annotation Pipeline, from our reference and other sequenced genomes and placed them into one file. Reference genomes included $B$. burgdorferi B31 (GCA_000008685.2), B. afzelii PKo (GCA 000222835.1), B. duttonii Ly (GCA_000019685.1), B. hermsii HS1 (GCA_001660005.1), B. miyamotoi CT132396 (GCA_001767415.1), B. miyamotoi FR64b (GCA 000568695.2), and the partially sequenced genome of Borrelia miyamotoi LB-2001 (GCA_000445425.4). (2) We clustered sequences using CD-HIT on a $90 \%$ level. (3) Each cluster's representative sequence was subjected to InterProScan analysis to determine whether it matches to a particular family of proteins in CDD, Pfam, or SUPERFAMILY database. (4) Subsequently, we extracted all sequences from the CD-HIT clusters which had their 
representative matched to specific IDs of specific PF. (5) Afterwards, we performed pairwise sequence alignment and distance tree reconstruction using a pairdist script [81] with 1000 bootstrap replicates which allowed us to understand the relatedness among specific PF genes from reference genomes with known plasmids names and the newly sequenced genome elements in our study. After designation of plasmid types the assembly of chromosome and plasmids were submitted to GenBank.

\section{Functional classification of proteins by comparison with previously defined COG}

Classification of proteins of the sequenced Izh-4 genome to clusters of orthologous groups (COG) was performed using a Perl script (cdd2cog.pl) from a collection of bacgenomics-scripts [82].

\section{Identification and phylogenetic analysis of $\mathrm{Vmp}$ genes}

As an independent and additional in silico analysis, we extracted all nucleotide sequences of ORFs (CDS and pseudogenes) from $B$. miyamotoi isolate Izh-4. We next subjected all these sequences to InterProScan analysis using the InterProScan match lookup service version 5.23-62.0, with a search against Pfam and SUPERFAMILY databases as an option. We subsequently retrieved all matches to PF01441, SSF63515 (Vsp proteins) or PF00921, SSF74748 (Vlp proteins) families. Finally, pairwise alignments of nucleotide sequences and phylogenetic analyses were performed using the pairdist script with 1000 bootstrap repetitions. The phylogenetic tree was visualized using Ete3 Python module.

\section{Phylogenies}

Identification of orthologous gene cluster and the production of a core genome alignment of chromosomes or particular plasmids was carried out using Roary v1.007002 [83]. For interspecies comparison among Borrelia chromosomes, a minimum of $70 \%$ identity for BLASTp searches was used, for intraspecies comparison of B. miyamotoi chromosomes this value was set to $95 \%$. A phylogenetic tree was inferred based on core genome alignments using RAxML v8.2.9 with GTR $+\Gamma$ nucleotide substitution model and 1000 bootstrap replicates. The phylogenetic tree was visualized using Python v2.7.11 and the Ete3 Python module.

\section{Supplementary information}

Supplementary information accompanies this paper at https://doi.org/10 1186/s12864-019-6388-4

Additional file 1: Table S1. Strains used in this study with NCBI

Biosample accession numbers.

Additional file 2: Method S1. Plasmid DNA separation by Pulsed-field Gel Electrophoresis (PFGE). Figures S1 - S6. Visual comparisons of ONT,
PacBio and final assemblies of plasmids of Borrelia miyamotoi isolate Izh4. Figures S7 - S15. Visual comparisons of contigs assembled from short Illumina reads for each PFGE fragment and final assemblies of plasmids of Borrelia miyamotoi isolate Izh-4. Figures S16 - S29. Visual comparisons of nucleotide sequences of plasmids of three B. miyamotoi strains CT132396, FR64b and Izh-4 to explore the similarity of plasmids.

Additional file 3: Figures $\mathbf{S 3 0}$ - $\mathbf{S 3 2}$ and Table S2. Nucleotide sequences of chromosomes of four B. miyamotoi genomes (3-USA, 1Japan) were aligned to the chromosome of Izh-4 by Mummer and positions of regions containing structural variation were detected by NucDiff and visualized in the IGV browser. Figure S33. Similarity of regions which contain the PF57/62 genes located on Ip18-1 and Ip18-2 plasmids of isolate Izh-4. Figure S34. Similarity of regions which contain PF57/62 genes located on Ip29 and Ip27 plasmids of Izh-4 isolate. Figure S35. Alignment of the intergenic region located upstream of the expressed Vmp gene on Ip41 of FR64b, Izh-4, CT13-2396, and LB-2001. Figure S36. Similarity of the right end of plasmids Ip41 and Ip23.

Additional file 4: Figure S37. PF32 phylogeny. Figure S38. PF49 phylogeny. Figure S39. PF50 phylogeny. Figure S40. PF57/62 phylogeny.

Additional file 5: Figure S41. Schematic dot plots of PacBio and ONT contigs with corresponding plasmid names aligned against itself.

\section{Abbreviations}

ANI: Average nucleotide identity; BLAST: Basic local alignment search tool; COG: Clusters of orthologous groups; cp: Circular plasmid; LB: Lyme borreliosis; Ip: Linear plasmid; ONT: Oxford nanopore technologies; ORF: Open reading frame; PF: Paralogous gene families of B. burgdorferi; Pfam: Protein family in protein families database (https://pfam.xfam.org/); PFGE: Pulsed-field gel electrophoresis; RF: Relapsing fever Borrelia; SMRT: Pacific bioscience single molecule real-time technology; SNP: Single nucleotide polymorphisms; Vlp: Variable large proteins; Vmp: Variable major protein; VNTR: Variable number tandem repeats; Vsp: Variable small proteins

\section{Acknowledgements}

The Pacific Biosciences sequencing service was provided by the Norwegian Sequencing Centre (www.sequencing.uio.no), a national technology platform hosted by the University of Oslo and supported by the "Functional Genomics" and "Infrastructure" programs of the Research Council of Norway and the Southeastern Regional Health Authorities.

\section{Authors' contributions}

AEP, JWH, GAS planned the study. JK, AEP, NMK, DSS performed Russian B. miyamotoi strain isolation and cultivation. IAG, NPK, MLM performed DNAlibrary preparation and whole-genome sequencing by Illumina. GM, VF, IAG provided PacBio whole-genome sequencing. KVK, GM, IAG, AEP performed PFGE analysis, comparative genomics and genome analysis. GM KVK AEP JWH VF GAS wrote the manuscript. All authors have read and approved the final manuscript.

\section{Funding}

This study was supported by the grant of Russian Science Foundation (project no. 15-15-00072-П) to AEP, KVK, NMK, IAG and DSS. The German National Reference Centre for Borrelia (VF) was funded by the Robert-KochInstitute, Berlin, Germany. JWH was supported by a grant from ZonMw (project number 522003007, Ticking on Pandora's box) and a grant from the EU Interreg North Sea Region program, as part of the NorthTick project.

\section{Availability of data and materials}

The datasets generated during the current study for Izh-4 isolate are available in the NCBI Sequence Read Archive (SRA) (www.ncbi.nlm.nih.gov/sra/). PacBio raw reads SRR7989200 (https://www.ncbi.nlm.nih.gov/sra/?term= SRR7989200), MinION raw reads SRR7989235 (https://www.ncbi.nlm.nih.gov/ sra/?term=SRR7989235), Illumina raw reads of total DNA-library SRR7989238 (https://www.ncbi.nlm.nih.gov/sra/?term=SRR7989238), Illumina raw reads for each PFGE fragments: N1 - SRR7989237 (https://trace.ncbi.nlm.nih.gov/ Traces/sra/?run=SRR7989237), N2 - SRR7989232 (https:/trace.ncbi.nlm.nih. gov/Traces/sra/?run=SRR7989232), N3 - SRR7989231 (https://trace.ncbi.nlm. nih.gov/Traces/sra/?run=SRR7989231), N4 - SRR7989234 (https://trace.ncbi. 
nlm.nih.gov/Traces/sra/?run=SRR7989234), N5 - SRR7989233 (https:/trace. ncbi.nlm.nih.gov/Traces/sra/?run=SRR7989233), N6 - SRR7989244 (https:// trace.ncbi.nlm.nih.gov/Traces/sra/?run=SRR7989244), N7 - SRR7989243 (https://trace.ncbi.nlm.nih.gov/Traces/sra/?run=SRR7989243), N8 - SRR7989198 (https://trace.ncbi.nlm.nih.gov/Traces/sra/?run=SRR7989198), N9 - SRR7989199 (https://trace.ncbi.nlm.nih.gov/Traces/sra/?run=SRR7989199).

The final set of chromosome and plasmids for Izn-4 isolate is available in the GenBank: chromosome - CP024390.1 (https://www.ncbi.nlm.nih.gov/nuccore/ CP024390), Ip72 - CP024391.1 (https://www.ncbi.nlm.nih.gov/nuccore/CP0243 91), Ip70 - CP024392.1 (https://www.ncbi.nlm.nih.gov/nuccore/CP024392.1), Ip64 - CP024401.2 (https://www.ncbi.nlm.nih.gov/nuccore/CP024401.2), Ip41 CP024393.1 (https://www.ncbi.nlm.nih.gov/nuccore/CP024393.1), cp30-1 CP024395.1 (https://www.ncbi.nlm.nih.gov/nuccore/CP024395.1), cp30-2 CP040828.1 (https://www.ncbi.nlm.nih.gov/nuccore/CP040828.1), lp29 CP024396.1 (https:/www.ncbi.nlm.nih.gov/nuccore/CP024396.1), Ip23 CP024397.1 (https://www.ncbi.nlm.nih.gov/nuccore/CP024397.1), Ip27 CP024398.1 (https://www.ncbi.nlm.nih.gov/nuccore/CP024398.1), Ip24 CP024399.2 (https://www.ncbi.n/m.nih.gov/nuccore/CP024399.2), Ip18-2 CP024400.2 (https://www.ncbi.nlm.nih.gov/nuccore/CP024400.2), Ip18-1 CP024405.2 (https://www.ncbi.n/m.nih.gov/nuccore/CP024405.2), Ip13 CP024404.1 (https://www.ncbi.nlm.nih.gov/nuccore/CP024404.1), lp6 CP024407.1 (https://www.ncbi.nlm.nih.gov/nuccore/CP024407.1).

\section{Ethics approval and consent to participate}

Not applicable.

\section{Consent for publication}

Not applicable.

\section{Competing interests}

The authors declare that they have no competing interests.

\section{Author details}

${ }^{1}$ Central Research Institute of Epidemiology, Moscow 111123, Russia. ${ }^{2}$ Federal State Budget Scientific Institution "Federal Scientific Center VIEV", Moscow, Russia. ${ }^{3}$ Bavarian Health and Food Safety Authority, German National Reference Centre for Borrelia, Veterinärstr. 2, 85764, Oberschleissheim, Germany. ${ }^{4}$ Amsterdam University Medical Centers, location Academic Medical Center, University of Amsterdam, Amsterdam, The Netherlands. ${ }^{5}$ Izmerov Research Institute of Occupational Health, Moscow, Russia. ${ }^{6}$ Chumakov Federal Scientific Center for Research and Development of Immune-and- Biological Products of Russian Academy of Sciences, Moscow, Russia. ${ }^{7}$ Izhevsk State Medical Academy, Izhevsk, Russia. ${ }^{8}$ Center of Strategical Planning and Management of Biomedical Health Risks of the Ministry of Health, Moscow, Russia.

\section{Received: 16 October 2019 Accepted: 12 December 2019}

Published online: 06 January 2020

\section{References}

1. Fukunaga M, Takahashi Y, Tsuruta Y, Matsushita O, Ralph D, McClelland M, et al. Genetic and phenotypic analysis of Borrelia miyamotoi sp. nov., isolated from the ixodid tick Ixodes persulcatus, the vector for Lyme disease in Japan. Int J Syst Bacteriol. 1995;45(4):804-10.

2. Cutler S, Vayssier-Taussat M, Estrada-Pena A, Potkonjak A, Mihalca AD, Zeller H. A new Borrelia on the block: Borrelia miyamotoi - a human health risk? Euro Surveill. 2019;24(18):1800170.

3. Platonov AE, Karan LS, Kolyasnikova NM, Makhneva NA, Toporkova MG, Maleev W, et al. Humans infected with relapsing fever spirochete Borrelia miyamotoi. Russia Emerg Infect Dis. 2011;17(10):1816-23.

4. Pukhovskaya NM, Morozova OV, Vysochina NP, Belozerova NB, Ivanov LI. Prevalence of Borrelia burgdorferi sensu lato and Borrelia miyamotoi in ixodid ticks in the Far East of Russia. Int J Parasitol Parasites Wildl. 2019;8: 192-202.

5. Iwabu-toh Y, Bazartseren B, Naranbaatar O, Yondonjamts E, Furuno K, Lee K, et al. Tick surveillance for Borrelia miyamotoi and phylogenetic analysis of isolates in Mongolia and Japan. Ticks Tick Borne Dis. 2017;8(6):850-7.

6. Rar V, Livanova N, Sabitova Y, Igolkina Y, Tkachev S, Tikunov A, et al. Ixodes persulcatus/pavlovskyi natural hybrids in Siberia: occurrence in sympatric areas and infection by a wide range of tick-transmitted agents. Ticks Tick Borne Dis. 2019;10(6):101254.
7. Khasnatinov MA, Danchinova GA, Takano A, Kawabata H, Ohashi N, Masuzawa T. Prevalence of Borrelia miyamotoi in Ixodes persulcatus in Irkutsk City and its neighboring territories, Russia. Ticks Tick Borne Dis. 2016; 7(2):394-7.

8. Krause PJ, Fish D, Narasimhan S, Barbour AG. Borrelia miyamotoi infection in nature and in humans. Clin Microbiol Infect. 2015;21(7):631-9.

9. Dibernardo A, Cote T, Ogden NH, Lindsay LR. The prevalence of Borrelia miyamotoi infection, and co-infections with other Borrelia spp. in Ixodes scapularis ticks collected in Canada. Parasit Vectors. 2014;7:183.

10. Ogden NH, Margos G, Aanensen DM, Drebot MA, Feil E, Hanincova K, et al. Investigation of genotypes of Borrelia burgdorferi in Ixodes scapularis ticks collected during surveillance in Canada. Appl Environ Microbiol. 2011;77(10):3244-54.

11. Kingry LC, Replogle A, Batra D, Rowe LA, Sexton C, Dolan M, et al. Toward a complete North American Borrelia miyamotoi genome. Genome Announc. 2017:5(5):e01557-16.

12. Fedorova N, Kleinjan JE, James D, Hui LT, Peeters H, Lane RS. Remarkable diversity of tick or mammalian-associated Borreliae in the metropolitan San Francisco Bay Area, California. Ticks Tick Borne Dis. 2014;5(6):951-61.

13. Padgett K, Bonilla D, Kjemtrup A, Vilcins IM, Yoshimizu MH, Hui L, et al. Large scale spatial risk and comparative prevalence of Borrelia miyamotoi and Borrelia burgdorferi sensu lato in Ixodes pacificus. PLoS One. 2014;9(10): e110853.

14. Kingry LC, Replogle A, Dolan M, Sexton C, Padgett KA, Schriefer ME, Chromosome and large linear plasmid sequences of a Borrelia miyamotoi strain isolated from Ixodes pacificus ticks from California. Genome Announc. 2017;5(37):e00960-17.

15. Cook VJ, Fedorova N, Macdonald WP, Lane RS, Barbour AG. Unique strain of Borrelia miyamotoi in Ixodes pacificus ticks, California, USA. Emerg Infect Dis. 2016;22(12):2205-7.

16. Wagemakers A, Staarink PJ, Sprong H, Hovius JW. Borrelia miyamotoi: a widespread tick-borne relapsing fever spirochete. Trends Parasitol. 2015; 31(6):260-9.

17. Geller J, Nazarova L, Katargina O, Jarvekulg L, Fomenko N, Golovljova I. Detection and genetic characterization of relapsing fever spirochete Borrelia miyamotoi in Estonian ticks. PLoS One. 2012;7(12):e51914.

18. Wagemakers A, Jahfari S, de Wever B, Spanjaard L, Starink MV, de Vries HJC et al. Borrelia miyamotoi in vectors and hosts in The Netherlands. Ticks Tick Borne Dis. 2017:8(3):370-4.

19. Blazejak K, Raulf MK, Janecek E, Jordan D, Fingerle V, Strube C. Shifts in Borrelia burgdorferi (s.l.) geno-species infections in Ixodes ricinus over a 10-year surveillance period in the city of Hanover (Germany) and Borrelia miyamotoispecific Reverse Line Blot detection. Parasit Vectors. 2018;11(1):304.

20. Palomar AM, Portillo A, Santibanez P, Santibanez S, Oteo JA. Borrelia miyamotoi: should this pathogen be considered for the diagnosis of tickborne infectious diseases in Spain? Enferm Infec Micr Cl. 2018;36(9):568-71.

21. Venczel R, Knoke L, Pavlovic M, Dzaferovic E, Vaculova T, Silaghi C, et al. A novel duplex real-time PCR permits simultaneous detection and differentiation of Borrelia miyamotoi and Borrelia burgdorferi sensu lato. Infection. 2016;44(1):47-55.

22. Crowder CD, Carolan HE, Rounds MA, Honig V, Mothes B, Haag H, et al. Prevalence of Borrelia miyamotoi in Ixodes ticks in Europe and the United States. Emerg Infect Dis. 2014;20(10):1678-82.

23. Barbour AG, Bunikis J, Travinsky B, Hoen AG, Diuk-Wasser MA, Fish D, et al. Niche partitioning of Borrelia burgdorferi and Borrelia miyamotoi in the same tick vector and mammalian reservoir species. Am J Trop Med Hyg. 2009;81(6):1120-31.

24. Salkeld DJ, Nieto NC, Bonilla DL, Yoshimizu MH, Padgett KA. Borrelia miyamotoi infections in small mammals, California, USA. Emerg Infect Dis. 2018;24(12):2356-9.

25. van Duijvendijk G, Coipan C, Wagemakers A, Fonville M, Ersoz J, Oei A, et al. Larvae of Ixodes ricinus transmit Borrelia afzelii and B. miyamotoi to vertebrate hosts. Parasit Vectors. 2016;9:97.

26. Han S, Lubelczyk C, Hickling GJ, Belperron AA, Bockenstedt LK, Tsao Jl. Vertical transmission rates of Borrelia miyamotoi in Ixodes scapularis collected from white-tailed deer. Ticks Tick Borne Dis. 2019;10(3):682-9.

27. Gofton AW, Margos G, Fingerle V, Hepner S, Loh SM, Ryan U, et al. Genomewide analysis of Borrelia turcica and 'Candidatus Borrelia tachyglossi' shows relapsing fever-like genomes with unique genomic links to Lyme disease Borrelia. Infect Genet Evol. 2018;66:72-81.

28. Barbour AG. Multiple and diverse vsp and vlp sequences in Borrelia miyamotoi, a hard tick-borne zoonotic pathogen. PLoS One. 2016;11(1):e0146283. 
29. Wagemakers A, Koetsveld J, Narasimhan S, Wickel M, Deponte K, Bleijlevens $B$, et al. Variable major proteins as targets for specific antibodies against Borrelia miyamotoi. J Immunol. 2016;196(10):4185-95.

30. Barbour AG, Burman N, Carter CJ, Kitten T, Bergstrom S. Variable antigen genes of the relapsing fever agent Borrelia hermsii are activated by promoter addition. Mol Microbiol. 1991;5(2):489-93.

31. Raffel SJ, Battisti JM, Fischer RJ, Schwan TG. Inactivation of genes for antigenic variation in the relapsing fever spirochete Borrelia hermsii reduces infectivity in mice and transmission by ticks. PLoS Pathog. 2014;10(4):e1004056.

32. Bunikis J, Tsao J, Garpmo U, Berglund J, Fish D, Barbour AG. Typing of Borrelia relapsing fever group strains. Emerg Infect Dis. 2004;10(9):1661-4.

33. Barbour AG. Phylogeny of a relapsing fever Borrelia species transmitted by the hard tick Ixodes scapularis. Infect Genet Evol. 2014;27:551-8.

34. Sarksyan DS, Platonov AE, Karan LS, Malinin IE, Khalitova LI, Shakhov VI, et al. Clinical presentation of "new" tick-borne borreliosis caused by Borrelia miyamotoi. Ter Arkh. 2012;84(11):34-41 (In Russ.)

35. Platonov AE, Toporkova MG, Kolyasnikova NM, Stukolova OA, Dolgova AS, Brodovikova AV, et al. Clinical presentation of Ixodes tick-borne borreliosis caused by Borrelia miyamotoi in the context of an immune response to the pathogen. Ter Arkh. 2017:89(11):35-43 (In Russ.).

36. Hovius JW, de Wever B, Sohne M, Brouwer MC, Coumou J, Wagemakers A, et al. A case of meningoencephalitis by the relapsing fever spirochaete Borrelia miyamotoi in Europe. Lancet. 2013;382(9892):658.

37. Gugliotta JL, Goethert HK, Berardi VP, Telford SR 3rd. Meningoencephalitis from Borrelia miyamotoi in an immunocompromised patient. N Engl J Med. 2013; 368(3):240-5.

38. Molloy PJ, Telford SR 3rd, Chowdri HR, Lepore TJ, Gugliotta JL, Weeks KE, et al. Borrelia miyamotoi disease in the Northeastern United States: a case series. Ann Intern Med. 2015;163(2):91-8.

39. Boden K, Lobenstein S, Hermann B, Margos G, Fingerle V. Borrelia miyamotoi-associated neuroborreliosis in immunocompromised person. Emerg Infect Dis. 2016;22(9):1617-20.

40. Henningsson AJ, Asgeirsson H, Hammas B, Karlsson E, Parke A, Hoornstra D, et al. Two cases of Borrelia miyamotoi meningitis, Sweden, 2018. Emerg Infect Dis. 2019;25(10):1965-8.

41. Hoornstra D, Koetsveld J, Sprong H, Platonov AE, Hovius JW. Borrelia miyamotoi disease in an immunocompetent patient, Western Europe. Emerg Infect Dis. 2018;24(9):1770-2.

42. Hue F, Ghalyanchi Langeroudi A, Barbour AG. Chromosome sequence of Borrelia miyamotoi, an uncultivable tick-borne agent of human infection. Genome Announc. 2013;1(5):e00713-13.

43. Margos G, Hepner S, Mang C, Marosevic D, Reynolds SE, Krebs S, et al. Lost in plasmids: next generation sequencing and the complex genome of the tick-borne pathogen Borrelia burgdorferi. BMC Genomics. 2017;18(1):422.

44. Kuleshov KV, Koetsveld J, Goptar IA, Markelov ML, Kolyasnikova NM, Sarksyan DS, et al. Whole-genome sequencing of six Borrelia miyamotoi clinical strains isolated in Russia. Genome Announc. 2018:6(1):e01424-17.

45. Walker BJ, Abeel T, Shea T, Priest M, Abouelliel A, Sakthikumar S, et al. Pilon: an integrated tool for comprehensive microbial variant detection and genome assembly improvement. PLoS One. 2014;9(11):e112963.

46. Casjens S, Murphy M, DeLange M, Sampson L, van Vugt R, Huang WM Telomeres of the linear chromosomes of Lyme disease spirochaetes: nucleotide sequence and possible exchange with linear plasmid telomeres. Mol Microbiol. 1997;26(3):581-96.

47. Huang WM, Robertson M, Aron J, Casjens S. Telomere exchange between linear replicons of Borrelia burgdorferi. J Bacteriol. 2004; 186(13):4134-41.

48. Tourand Y, Kobryn K, Chaconas G. Sequence-specific recognition but position-dependent cleavage of two distinct telomeres by the Borrelia burgdorferi telomere resolvase, ResT. Mol Microbiol. 2003;48(4):901-11.

49. Tourand Y, Deneke J, Moriarty TJ, Chaconas G. Characterization and in vitro reaction properties of 19 unique hairpin telomeres from the linear plasmids of the Lyme disease spirochete. J Biol Chem. 2009;284(11):7264-72.

50. Moriarty TJ, Chaconas G. Identification of the determinant conferring permissive substrate usage in the telomere resolvase, ResT. J Biol Chem. 2009;284(35):23293-301.

51. Casjens SR, Mongodin EF, Qiu WG, Luft BJ, Schutzer SE, Gilcrease EB, et al. Genome stability of Lyme disease spirochetes: comparative genomics of Borrelia burgdorferi plasmids. PLoS One. 2012;7(3):e33280.

52. Chaconas G, Kobryn K. Structure, function, and evolution of linear replicons in Borrelia. Annu Rev Microbiol. 2010;64:185-202.
53. Stevenson B, Zuckert WR, Akins DR. Repetition, conservation, and variation: the multiple cp32 plasmids of Borrelia species. J Mol Microbiol Biotechnol. 2000;2(4):411-22.

54. Casjens S, van Vugt R, Tilly K, Rosa PA, Stevenson B. Homology throughout the multiple 32-kilobase circular plasmids present in Lyme disease spirochetes. J Bacteriol. 1997;179(1):217-27.

55. Eggers $\mathrm{CH}$, Samuels DS. Molecular evidence for a new bacteriophage of Borrelia burgdorferi. J Bacteriol. 1999;181(23):7308-13.

56. Fraser CM, Eisen JA, Nelson KE, Paulsen IT, Salzberg SL. The value of complete microbial genome sequencing (you get what you pay for). J Bacteriol. 2002;184(23):6403-5.

57. Loman NJ, Quick J, Simpson JT. A complete bacterial genome assembled de novo using only nanopore sequencing data. Nat Methods. 2015;12(8): 733-5.

58. Ring N, Abrahams JS, Jain M, Olsen H, Preston A, Bagby S. Resolving the complex Bordetella pertussis genome using barcoded nanopore sequencing. Microb Genom. 2018:4(11):e000234.

59. Wick RR, Judd LM, Gorrie CL, Holt KE. Completing bacterial genome assemblies with multiplex MinION sequencing. Microb Genom. 2017:3(10): e000132.

60. Casjens SR, Di L, Akther S, Mongodin EF, Luft BJ, Schutzer SE, et al. Primordial origin and diversification of plasmids in Lyme disease agent bacteria. BMC Genomics. 2018;19(1):218.

61. Casjens SR, Gilcrease EB, Vujadinovic M, Mongodin EF, Luft BJ, Schutzer SE, et al. Plasmid diversity and phylogenetic consistency in the Lyme disease agent Borrelia burgdorferi. BMC Genomics. 2017;18(1):165.

62. Koetsveld J, Kolyasnikova NM, Wagemakers A, Toporkova MG, Sarksyan DS, Oei A, et al. Development and optimization of an in vitro cultivation protocol allows for isolation of Borrelia miyamotoi from patients with hard tick-borne relapsing fever. Clin Microbiol Infect. 2017;23(7):480-4.

63. Ribot EM, Fair MA, Gautom R, Cameron DN, Hunter SB, Swaminathan B, et al. Standardization of pulsed-field gel electrophoresis protocols for the subtyping of Escherichia coli O157:H7, Salmonella, and Shigella for PulseNet. Foodborne Pathog Dis. 2006;3(1):59-67.

64. BBTools. Joint Genome Institute. https://jgi.doe.gov/data-and-tools/bbtools/. Accessed 9 Oct 2019.

65. Bankevich A, Nurk S, Antipov D, Gurevich AA, Dvorkin M, Kulikov AS, et al. SPAdes: a new genome assembly algorithm and its applications to singlecell sequencing. J Comput Biol. 2012;19(5):455-77.

66. Porechop: adapter trimmer for Oxford Nanopore reads. https://github.com/ rrwick/Porechop. Accessed 9 Oct 2019.

67. FlexiDot: dotplots for visual sequence analyses. https://github.com/molbiodresden/flexidot. Accessed 9 Oct 2019.

68. Katoh K, Standley DM. MAFFT multiple sequence alignment software version 7: improvements in performance and usability. Mol Biol Evol. 2013; 30(4):772-80.

69. APC: (a) (p) erfect (c) ircle https://github.com/jfass/apc. Accessed 9 Oct 2019.

70. Nanopolish: signal-level algorithms for MinION data. https://github.com/jts/ nanopolish. Accessed 9 Oct 2019.

71. Rice $\mathrm{P}$, Longden I, Bleasby A. EMBOSS: the European molecular biology open software suite. Trends Genet. 2000;16(6):276-7.

72. Python module for average nucleotide identity analyses. https://github. com/widdowquinn/pyani. Accessed 9 Oct 2019.

73. Circos: circular visualization. http://circos.ca/. Accessed 9 Oct 2019

74. Delcher AL, Salzberg SL, Phillippy AM. Using MUMmer to identify similar regions in large sequence sets. Current protocols in bioinformatics. 2003; Chapter 10: Unit 10.3.

75. Khelik K, Lagesen K, Sandve GK, Rognes T, Nederbragt AJ. NucDiff: in-depth characterization and annotation of differences between two sets of DNA sequences. BMC Bioinformatics. 2017;18(1):338.

76. Sullivan MJ, Petty NK, Beatson SA. Easyfig: a genome comparison visualizer. Bioinformatics. 2011;27(7):1009-10.

77. NCBI Prokaryotic Genome Annotation Pipeline. https://github.com/ncbi/ pgap. Accessed 9 Oct 2019.

78. Marchler-Bauer A, Derbyshire MK, Gonzales NR, Lu S, Chitsaz F, Geer LY, et al. CDD: NCBI's conserved domain database. Nucleic Acids Res. 2015; 43(Database issue):D222-6.

79. El-Gebali S, Mistry J, Bateman A, Eddy SR, Luciani A, Potter SC, et al. The Pfam protein families database in 2019. Nucleic Acids Res. 2019:47(D1): D427-D32. 
80. Pandurangan AP, Stahlhacke J, Oates ME, Smithers B, Gough J. The SUPERFAMILY 2.0 database: a significant proteome update and a new webserver. Nucleic Acids Res. 2019;47(D1):D490-D4.

81. PairDist. https://github.com/frederic-mahe/pairdist.git. Accessed 9 Oct 2019.

82. Collection of scripts for bacterial genomics. https://github.com/aleimba/bacgenomics-scripts. Accessed 9 Oct 2019.

83. Page AJ, Cummins CA, Hunt M, Wong VK, Reuter S, Holden MT, et al. Roary: rapid large-scale prokaryote pan genome analysis. Bioinformatics. 2015; 31(22):3691-3

\section{Publisher's Note}

Springer Nature remains neutral with regard to jurisdictional claims in published maps and institutional affiliations.

Ready to submit your research? Choose BMC and benefit from:

- fast, convenient online submission

- thorough peer review by experienced researchers in your field

- rapid publication on acceptance

- support for research data, including large and complex data types

- gold Open Access which fosters wider collaboration and increased citations

- maximum visibility for your research: over $100 \mathrm{M}$ website views per year

At $\mathrm{BMC}$, research is always in progress.

Learn more biomedcentral.com/submissions 